Commun. Math. Phys. 141, 79-117 (1991)

\title{
Computer Calculation of Witten's 3-Manifold Invariant*
}

\author{
Daniel S. Freed and Robert E. Gompf \\ Department of Mathematics, University of Texas at Austin, Austin, TX 78712, USA
}

Received November 5, 1990; in revised form March 21, 1991

\begin{abstract}
Witten's $2+1$ dimensional Chern-Simons theory is exactly solvable. We compute the partition function, a topological invariant of 3-manifolds, on generalized Seifert spaces. Thus we test the path integral using the theory of 3manifolds. In particular, we compare the exact solution with the asymptotic formula predicted by perturbation theory. We conclude that this path integral works as advertised and gives an effective topological invariant.
\end{abstract}

Quantum Field Theory is rapidly emerging as the unifying principle behind new topological invariants in low dimensions. Ed Witten led this development with his introduction of topological quantum field theories. From many points of view the most accessible of these quantum theories is the $2+1$ dimensional Chern-Simons theory [W]. There is a beautiful corresponding classical theory [F1] defined for each class in $H^{4}(B G)$, where $G$ is a compact Lie group and $B G$ its classifying space. In case $G$ is finite the quantization is straightforward and illuminating [DW, FQ], though the topological invariants arising from the quantum theory are trivial. On the other hand the quantization of the theory for continuous groups proceeds via the Feynman path integral, which is not (yet) a mathematically rigorous procedure. Nevertheless, this quantum theory (presuming it exists) reproduces the Jones polynomial of links in $S^{3}$ and generates new invariants of links in arbitrary closed oriented 3-manifolds. Some of Witten's assertions in [W] now have mathematical proofs independent of path integral arguments. We give evidence of a different kind for the validity of the quantum theory - computer calculations.

In this paper we restrict our attention to the simplest continuous group $G=S U(2)$. Then $H^{4}(B G) \cong \mathbb{Z}$, so there is an $S U(2)$ theory for each integer $k$,

\footnotetext{
* The first author is supported by NSF grant DMS-8805684, an Alfred P. Sloan Research Fellowship, and a Presidential Young Investigators award. The second author is supported by NSF grant DMS-8902153
} 
typically called the level. A nondegeneracy requirement forbids $k=0$, while theories at opposite integers are trivially related, at least on the classical level. Thus we restrict $k$ to be a positive integer. In Sect. 1 we summarize the relevant information in $[\mathrm{W}]$. Here we review both the exact solution and the perturbation theory for large $k^{1}$ Witten's invariant depends on a certain framing of the 3manifold; we use the canonical 2-framing of Atiyah [A1]. The Kirby calculus keeps track of these framings nicely. The primary new theoretical contribution of this paper is the explicit computation of these framings, as we explain in detail in Sect. 2. We also record various topological invariants of lens spaces and Brieskorn homology spheres. As we ask the reader to trust our computer code, we present some brief evidence for the validity of our programs in Sect. 3. The computations are tabulated, graphed, and analyzed in Sect. 4 . We test the exact solution in various ways and then compare the exact solution to the perturbation theory. We summarize our findings in three words: It all works! There are a few details that we could not resolve. We list them in Sect. 5 together with some conclusions and open questions.

Physicists have great confidence in the path integral based on theoretical work and on their experience in the lab. Measurements of physical quantities such as the anomalous magnetic dipole moment of the electron agree with perturbative calculations from path integrals to remarkable accuracy. Many mathematicians, however, remain incredulous. Lacking a rigorous justification for path integral manipulations, they often cast a skeptical eye on physicist's methods and results. Topological Quantum Field Theory not only illuminates invariants in low dimensional topology, but also provides a new testing ground for the path integral. The Chern-Simons theory discussed in this paper is unusual among quantum field theories in dimensions $\geqq 3-$ it is exactly solvable. Hence we can test the gluing law, ${ }^{2}$ the fundamental property of the path integral, using the exact solution. Furthermore, as with any quantum field theory there is a perturbative expansion for the path integral - here for large $k$ - and we can test the exact solutions against this standard perturbative expansion. In our experiments the standards against which we measure the path integral calculations are not experiments in a lab, but rather the mathematical theory of 3-manifolds. It is worth emphasizing how different is the mathematics used to derive the formulas for the exact solution (symplectic geometry, Kač-Moody Lie algebras) from the mathematics used to derive the perturbative expansion (gauge theory, Gaussian measures). Also, it is the locality of the integral which implies the gluing law, not the mathematics of the exact solution. The Chern-Simons path integral passed all of our tests with flying colors. Not only were manipulations intrinsic to the quantum theory tested, but the topological predictions that came out were verified by a mathematical theory completely extrinsic to any quantum considerations. We hope that these results will inspire increased belief in Quantum Field Theory - yes, it is to some extent still a religion - and in its potential contributions to geometry and to mathematics generally.

Our work can be extended in several directions. First, we only carry out computations for the group $S U(2)$, and one might hope to learn more from other

\footnotetext{
1 We correct a slight error in Witten's asymptotic formula

2 The path integral over a manifold whose boundary on one side is $Y$ and on the other is $Y^{\prime}$ should be viewed as an evolution from the Hilbert space of states on $Y$ to the Hilbert space of states on $Y^{\prime}$. The gluing law (1.12), which is the Euclidean field theoretic version of the group law in quantum mechanics, states that these evolutions compose when we glue manifolds together
} 
Lie groups. In addition, the specific computations we make only use the simplest data from the associated conformal field theory: the central charge, the conformal weights, and the " $S$ matrix." More interesting examples, particularly hyperbolic 3manifolds, can be treated using the "braiding matrices." We hope to extend our computations in future work.

After we carried out most of our computations, Paul Melvin pointed out that our formula for Witten's invariant of a Seifert manifold - the right-hand side of (1.17) - can be shown to equal the invariant of Reshetikhin and Turaev [RT]. This equality follows from [KM2, Theorem 3.27]. Since Reshetikhin and Turaev prove that their construction gives a topological invariant, this provides a rigorous proof for some of our observations about Witten's exact solution. Recent work of Walker [Wa], based in part on the ideas of Reshetikhin and Turaev, goes further he constructs the complete $S U(2)$ Chern-Simons theory and proves the gluing law. However, we do not know of any rigorous derivation of the asymptotic expansion for large $k$. Our numerical data remains the only evidence that this expansion is valid. In addition, our calculations for finite $k$ illustrate the effectiveness of Witten's invariant.

Karen Uhlenbeck provided not only most of the computer equipment, but also much inspiration and advice. We also thank Rob Kirby, Lisa Jeffrey, Paul Melvin, Danny Ruberman, Ron Stern, and Kevin Walker for useful conversations. Ed Witten answered some of the questions we raised in a preliminary version of this paper. The referee's comments improved the exposition in several places. The first author warmly thanks the Aspen Center for Physics for its hospitality during the final stages of this work.

\section{Chern-Simons Theory}

The classical Chern-Simons action [F1] is a function of a connection over a 3manifold $X$. Since all $S U(2)$ bundles are trivializable over $X$, we may take the connection to be an $\mathfrak{s u}(2)$-valued 1-form $A$ on $X$. Then the action is ${ }^{3}$

$$
S_{X}(A)=\frac{1}{8 \pi^{2}} \int_{X} \operatorname{Tr}\left(A \wedge d A+\frac{2}{3} A^{3}\right) .
$$

This is well-defined in $\mathbb{R} / \mathbb{Z}$ if $X$ is closed and oriented. Note that $S_{X}(A)$ changes sign if the orientation of $X$ is reversed. The path integral ${ }^{4}$ at level $k$ is then

$$
Z_{X}(k)=Z_{X}=\int d A e^{2 \pi i k S_{X}(A)},
$$

where the integration is over all gauge equivalence classes of connections. No measure $d A$ has been constructed as we write, so we must regard (1.2) as a formal expression. Nonetheless, Witten gives an explicit recipe for computing $Z_{X}$, based ultimately on formulas (1.7) and (1.8) from the theory of Kač-Moody Lie algebras. It is important to realize, though, that the recipe also uses geometric properties of the path integral, not just these algebraic formulae. We briefly review his results and derive a formula for a specific class of 3-manifolds, the Seifert fibered spaces.

\footnotetext{
${ }^{3}$ Our normalization differs from Witten's by a factor of $2 \pi$

4 Our mathematical readers may wish to consult [A2] for a general description of topological quantum field theory
} 
Then we discuss the large $k$ asymptotics of (1.2), which is derived from standard path integral arguments.

Exact Solution. Let $X$ be a closed oriented 3-manifold. Then the exact solution for the path integral (1.2) depends on a framing of twice the tangent bundle as a Spin(6) bundle, henceforth referred to as a 2-framing. Atiyah [A1] has shown that there is a canonical choice $\alpha$ for this 2 -framing. It is characterized by the following property: If $W$ is an oriented 4-manifold with boundary $X$, then the signature of $W$ is

$$
\sigma(W)=\frac{1}{6} p_{1}(2 T(W), \alpha),
$$

where the relative Pontrjagin class is computed using the framing $\alpha$ on the boundary. A change of framing by one unit multiplies the path integral $Z_{X}$ by the factor

$$
e^{-2 \pi i c / 24} \text {, }
$$

where $c$ is the central charge. For the $S U(2)$ theory at level $k$, we have

$$
c=\frac{3 k}{k+2} \text {. }
$$

A key ingredient in our work is the explicit computation of the canonical 2-framing, which we undertake in Sect. 2.

The quantum field theory assigns a finite dimensional complex inner product space $E$ to the standard torus $T^{2}=S^{1} \times S^{1}$. At level $k$ it has dimension $k+1$ and a distinguished basis which we denote $1,2, \ldots, \mathbf{k}+\mathbf{1}$. The inner product is

$$
\langle\boldsymbol{\alpha}, \boldsymbol{\beta}\rangle=\delta_{\alpha \beta} \text {. }
$$

We use this inner product to raise and lower indices. The vector space $E$ carries a unitary representation of $S L(2 ; \mathbb{Z})$. The standard generators

$$
T=\left(\begin{array}{ll}
1 & 1 \\
0 & 1
\end{array}\right) \text { and } S=\left(\begin{array}{rr}
0 & -1 \\
1 & 0
\end{array}\right)
$$

act by the matrices

$$
\begin{gathered}
\tilde{T}_{\beta}^{\alpha}=e^{2 \pi i\left(h_{\alpha}-c / 24\right)} \delta_{\beta}^{\alpha}, \\
\tilde{S}_{\beta}^{\alpha}=\sqrt{\frac{2}{k+2}} \sin \left(\frac{\alpha \beta \pi}{k+2}\right),
\end{gathered}
$$

where $\alpha, \beta=1,2, \ldots, \mathrm{k}+1$ and $h_{\alpha}$ is the conformal weight

$$
h_{\alpha}=\frac{\alpha^{2}-1}{4(k+2)} \text {. }
$$

More generally, the theory attaches a hermitian vector space $E(Y)$ to each (stably 2-framed) closed oriented 2-manifold $Y$ and a vector $Z_{X} \in E(\partial X)$ to each (2-framed) compact oriented 3-manifold $X$. For example, the vector attached to the solid torus $D^{2} \times S^{1}$ is $1 \in E(\partial X)=E$. The theory also assigns vectors to framed ${ }^{5}$ oriented links in 3-manifolds if each component of the link is labeled with a basis element of $E$. For example, the vector attached to the single component link representing $p t \times S^{1}$ is $\alpha \in E$, if that link has label $\alpha$. These vector spaces and vectors obey

5 The framing here is a trivialization of the normal bundle to the link 
properties easily derived from the path integral. ${ }^{6}$ Thus if $-X$ denotes $X$ with the opposite orientation, then

$$
Z_{-X}=\overline{Z_{X}}
$$

Also, if $X_{1} \# X_{2}$ is the connected sum, then ${ }^{7}$

$$
Z_{X_{1} \# X_{2}}=\frac{Z_{X_{1}} Z_{X_{2}}}{Z_{S^{3}}}
$$

Fig. 1

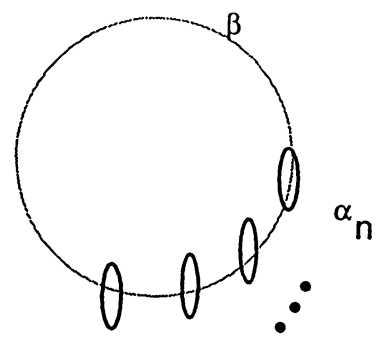

Other properties are the multiplicativity under disjoint union and the gluing law. The latter states that if $Y \subset X$ is an oriented codimension one submanifold, and $X^{\text {cut }}$ is the manifold obtained by cutting $X$ along $Y$, then

$$
Z_{X}=\operatorname{Tr}_{Y}\left(Z_{X^{\text {cut }}}\right)
$$

where $\operatorname{Tr}_{Y}$ is the inner product

$$
\operatorname{Tr}_{Y}: E\left(\partial X^{\mathrm{cut}}\right) \cong E(\partial X) \otimes E(Y) \otimes \overline{E(Y)} \rightarrow E(\partial X)
$$

over the state space of $Y$. We use these properties freely in the derivations which follow.

First, since $S^{3}$ is obtained by gluing two solid tori along their common boundary after twisting one of the boundaries by the matrix $S$, we have $[\mathrm{W},(4.38)]$

$$
Z_{S^{3}}=\langle\tilde{S}(\mathbf{1}), \mathbf{1}\rangle=\tilde{S}_{11}=\sqrt{\frac{2}{k+2}} \sin \left(\frac{\pi}{k+2}\right) .
$$

Similarly, for the Hopf link in $S^{3}$ with labels $\alpha, \beta$, the invariant is $\widetilde{S}_{\alpha \beta}[\mathrm{W},(4.47)]$. Now using the connected sum formula $[\mathrm{W},(4.6)]$ we compute the invariant for the link shown in Fig. 1 to be

$$
\frac{\widetilde{S}_{\alpha_{1} \beta} \tilde{S}_{\alpha_{2} \beta} \ldots \tilde{S}_{\alpha_{n} \beta}}{\left(\widetilde{S}_{1 \beta}\right)^{n-1}} .
$$

If $n=3$, then (1.14) reduces to a formula of Verlinde [V, W, Fig. 17b].

\footnotetext{
${ }^{6}$ Atiyah [At] axiomatizes these properties. They are used by Witten in [W, Sect. 4], and our formulae below are simply derived from his work there

7 Equation (1.11) holds for any given 2-framings on $X_{1}$ and $X_{2}$, the induced 2-framing on $X_{1} \# X_{2}$, and the canonical 2-framing on $S^{3}$. The existence of a well-defined induced 2-framing on $X_{1} \# X_{2}$ follows from the uniqueness (up to isotopy) of orientation preserving selfdiffeomorphisms of $S^{2}$. (Compare this with our discussion below of gluing along a torus.) The canonical 2-framings on $X_{1}$ and $X_{2}$ induce the canonical 2-framing on $X_{1} \# X_{2}$, by (1.3)
} 
We compute the Witten invariant for lens spaces and Seifert fibered spaces, though our methods apply to the larger class of manifolds obtained by plumbing on trees. Let $p_{1} / q_{1}, \ldots, p_{n} / q_{n}$ be rational numbers in lowest terms. The Seifert manifold $^{8}$

$$
X=X\left(p_{1} / q_{1}, \ldots, p_{n} / q_{n}\right)
$$

is obtained from $S^{2} \times S^{1}$ by removing $n$ disjoint solid tori $D_{i} \times S^{1}\left(D_{1}, \ldots, D_{n}\right.$ disjoint disks in $S^{2}$ ) and gluing them back after twisting the boundary by certain $S L(2 ; \mathbb{Z})$ matrices $M_{1}, \ldots, M_{n}$. The matrix $M_{i}=M\left(p_{i}, q_{i}\right)$ has ${ }^{9}$ first column $\left(\begin{array}{l}p_{i} \\ q_{i}\end{array}\right)$. The particular choice of $M_{i}$ among all such matrices affects the 2-framing, but not the diffeomorphism type of the resulting manifold; we specify our choice below (2.2). Let $\tilde{M}_{i}$ denote the corresponding transformation of $E$. The gluing law implies

$$
Z_{X}=\left\langle\tilde{M}_{1}(1) \otimes \ldots \otimes \tilde{M}_{n}(1), \psi\right\rangle,
$$

where the inner product takes place in $E^{\otimes n}$, and $\psi$ is the path integral over $S^{2} \times S^{1}$ with the $n$ solid tori deleted. Expanding, we have

$$
Z_{X}=\sum_{\alpha_{1}, \ldots, \alpha_{n}}\left(\tilde{M}_{1}\right)_{1}^{\alpha_{1}} \ldots\left(\tilde{M}_{n}\right)_{1}^{\alpha_{n}}\left\langle\alpha_{1} \otimes \ldots \otimes \alpha_{n}, \psi\right\rangle
$$

The last factor is the partition function of $S^{2} \times S^{1}$ with an $n$ component link of disjoint circles $p t \times S^{1}$ labeled $\alpha_{1}, \ldots, \alpha_{n}$; it is the dimension of the vector space attached to $S^{2}$ with $n$ labeled punctures. We compute it via surgery on a parallel circle, which changes $S^{2} \times S^{1}$ into $S^{3}$ and converts the $n+1$ parallel circles into the link depicted in Fig. 1. So from (1.16) and (1.14) we obtain

$$
Z_{X}=\sum_{\alpha_{1}, \ldots, \alpha_{n}, \beta}\left(\tilde{M}_{1}\right)_{1}^{\alpha_{1} \ldots}\left(\tilde{M}_{n}\right)_{1}^{\alpha_{n}} \frac{\tilde{S}_{\alpha_{1} \beta} \tilde{S}_{\alpha_{2} \beta} \ldots \tilde{S}_{\alpha_{n} \beta}}{\left(\widetilde{S}_{1 \beta}\right)^{n-2}}
$$

This is our basic formula. The lens space $L(p, q)$ is the Seifert manifold

$$
L(p, q)=X(q / p) \text {. }
$$

In this case the invariant is easy to compute directly as

$$
Z_{L(p, q)}=(\tilde{M}(q, p))_{1}^{1} \text {. }
$$

In these formulas we have not kept track of the 2-framings. We postpone those considerations until Sect. 2.

Suppose $L$ is a link in $S^{\mathbf{3}}$, and we label each component of $L$ with 2 . Then Witten shows [W, Sect. 4.1] that the path integral here reproduces a certain value of the Jones polynomial. Following [KM1] we let $\widetilde{V}_{L}(t)$ be the version of the Jones polynomial satisfying the skein relation

$$
t \widetilde{V}_{L_{+}}(t)-\left(t^{1 / 2}-t^{-1 / 2}\right) \tilde{V}_{L_{0}}-t^{-1} \tilde{V}_{L_{-}}(t)=0
$$

and normalized by $\widetilde{V}_{\text {unknot }}(t)=1$. Then for the left-handed trefoil knot, for example, we have

$$
\widetilde{V}_{\text {tref }}(t)=-t^{4}+t^{3}+t
$$

\footnotetext{
8 Usually the term "Seifert manifold" is only used when all $p_{i} / q_{i}$ are nonzero. However, our methods also apply when some $p_{i}$ or $q_{i}$ are zero. For example, any $p_{i} / q_{i}=1 / 0$ may be deleted without changing the manifold

9 The first basis element is the meridian $\partial D_{i} \times p t C \partial D_{i} \times S^{1}$. The second basis element is the longitude $p t \times S^{1} \subset \partial D_{i} \times S^{1}$
} 
Witten's invariant $Z_{\left(S^{3} ; L\right)}$ for the link $L$ depends on a choice of normal framing (framing of the normal bundle) for $L$. Adding a right-handed twist to the normal framing multiplies $Z_{\left(S^{3} ; L\right)}$ by

$$
e^{2 \pi i\left(\frac{3}{4(k+2)}\right.}
$$

The invariant becomes well-defined if we agree to use a framing for which a parallel push-off of $L$ has linking number 0 with $L .{ }^{10}$ Since Witten's invariant for an unknown is $\tilde{S}_{12}$, for a link $L \subset S^{3}$ with label 2 we obtain

$$
Z_{\left(S^{3} ; L\right)}=\sqrt{\frac{2}{k+2}} \sin \left(\frac{2 \pi}{k+2}\right) \tilde{V}_{L}\left(e^{2 \pi i /(k+2)}\right) .
$$

Let $M$ denote a matrix in $S L(2 ; \mathbb{Z})$ and $\tilde{M}$ the corresponding transformation of $E$. Then $M$ determines an oriented 3-manifold $X_{M}$ which fibers over $S^{1}$, namely the mapping torus determined by the action of $M$ on $T^{2}$. Up to a correction for the framing, the partition function can be computed from the gluing law as

$$
Z_{X_{M}}=\operatorname{Trace} \tilde{M} \text {. }
$$

Perturbation Theory. Standard path integral techniques predict the behavior of $Z_{X}(k)$ for large $k$ [W, Sect. 2]. By stationary phase the leading order contributions comes from the classical solutions, i.e., the stationary points of the action (1.1). The Euler-Lagrange equation

$$
d A+A^{2}=0
$$

asserts that the classical solutions are connections with vanishing curvature. Recall that these flat connections are determined up to gauge equivalence by a representation $\pi_{1}(\mathrm{X}) \rightarrow \mathrm{SU}(2)$ up to conjugacy (if $X$ is connected). For now assume that there is a finite number of gauge equivalence classes of solutions to (1.25), represented by a finite set of flat connections $\left\{A_{i}\right\}$. For any flat connection $A$ we form the elliptic complex

$$
0 \longrightarrow \Omega_{X}^{0}(\mathfrak{s u}(2)) \stackrel{d_{A}}{\longrightarrow} \Omega_{X}^{1}(\mathfrak{s u}(2)) \stackrel{d_{A}}{\longrightarrow} \Omega_{X}^{2}(\mathfrak{s u}(2)) \stackrel{d_{A}}{\longrightarrow} \Omega_{X}^{3}(\mathfrak{s u}(2)) \longrightarrow 0
$$

where $d_{A}$ is the covariant derivative in the adjoint representation. Since $A$ is flat, we have $d_{A}^{2}=0$. Denote the cohomology groups of $(1.26)$ by $H_{X}^{\circ}(A)$. The hypothesis that the gauge equivalence class of $A$ is isolated implies $H_{X}^{1}(A)=0$. Assume also $H_{X}^{0}(A)=0$, i.e., assume that $A$ is an irreducible connection. By Poincaré duality we also have $H_{X}^{2}(A)=H_{X}^{3}(A)=0$. Since all of the cohomology vanishes, the Reidemeister torsion $\tau_{X}(A) \in \mathbb{R}^{+}$is well-defined [ $\left.\mathrm{Re}, \mathrm{Mi}\right]$. The torsion does not depend on the orientation of $X$. It enters the asymptotic calculation in another form, the analytic torsion [RS1], which is a certain ratio of determinants of Laplacians. The equality of analytic torsion and Reidemeister torsion was proved by Cheeger [C] and Müller [Mü]. If $H_{X}^{\bullet}(A) \neq 0$ then the torsion is defined once we choose a basis for the cohomology $H_{X}^{\circ}(A)$. [In fact, it only depends on the induced volume element in $\otimes\left(\operatorname{det} H_{X}^{i}(A)\right)^{\otimes(-1)^{i}}$.]

Another topological invariant of a flat connection $A$ which appears in the asymptotic formula is the spectral flow $I_{A}$ from the trivial connection to $A$. This is

${ }^{10}$ For knots this is the 0 -framing. For links we choose any framing for which the sum of all the entries of the linking matrix is 0 
the same spectral flow which appears in Floer homology [Fl, FS]. It arises in the asymptotic formula through the $\eta$-invariant of Atiyah, Patodi, and Singer [APS]. Choose a Riemannian metric on $X$, and consider the self-adjoint operator

$$
(-1)^{p}\left(* d_{A}+d_{A} *\right)
$$

acting on $\oplus_{p=0}^{1} \Omega_{X}^{2 p+1}(\mathfrak{s u}(2))$. Let $\eta(A) \in \mathbb{R}$ denote its $\eta$-invariant. ${ }^{11}$ If $\theta$ denotes the trivial connection, then the difference

$$
\varrho(A)=\eta(A)-\eta(\theta)
$$

is a topological invariant independent of the metric on $X$. Its reduction $(\bmod 1)$ is related to the Chern-Simons invariant $S_{X}(A)$ via the formula

$$
\varrho(A)=16 S_{X}(A) \quad(\bmod 1) .
$$

[This follows from (1.31) below.] The fact that $\varrho(A)$ is defined over the reals allows us to rewrite it in terms of the spectral flow. Recall that the self-dual complex of a Riemannian 4-manifold is the elliptic complex

$$
0 \longrightarrow \Omega_{W}^{0} \stackrel{d}{\longrightarrow} \Omega_{W}^{1} \stackrel{d-}{\longrightarrow} \Omega_{W}^{2,-} \longrightarrow 0,
$$

where $\Omega_{W}^{2,-}$ are the anti-self-dual 2-forms on $W$. Fix a metric on $X$ and consider the induced product metric on the cylinder $W=X \times[0,1]$. Choose a path of connections from the trivial connection $\theta$ to $A$, which then forms a connection over $W$. Now couple the self-duality operator associated to the complex (1.30) to this connection and impose the Atiyah-Patodi-Singer boundary conditions. The index $I_{A}$ of this operator (taken modulo 8) is the spectral flow we seek; it is independent of the metric on $X$ and the path of connections. In general it is related to (1.28) by the formula ${ }^{12}$

$$
\frac{I_{A}}{4}=2 S_{X}(A)-\frac{\varrho(A)}{8}-\frac{3\left(1+b^{1}(X)\right)}{8}-\frac{\operatorname{dim} H_{X}^{0}(A)+\operatorname{dim} H_{X}^{1}(A)}{8} \quad(\bmod 2),
$$

where $b^{1}(X)$ is the first Betti number of $X$. Equation (1.31) follows from the index theorem in [APS(i)] applied to $W$. If we reverse the orientation of $X$, then $\varrho(A)$ and $S_{X}(A)$ change sign; therefore, $I_{A}$ changes in a manner dictated by (1.31).

Now we state the perturbation theory result. Recall we are assuming that there is a finite set of gauge equivalence classes of flat connections over $X$, represented by $\left\{A_{i}\right\}$, and that the cohomology $H_{X}^{\bullet}\left(A_{i}\right)$ vanishes. It follows that $b^{1}(X)=0$ also. Then

$$
Z_{X}(k) \sim \frac{1}{2} e^{-3 \pi i / 4} \sum_{i} \sqrt{\tau_{X}\left(A_{i}\right)} e^{-2 \pi i I_{A_{i}} / 4} e^{2 \pi i S_{X}\left(A_{i}\right)(k+2)}
$$

\footnotetext{
${ }^{11}$ It is defined whether or not $A$ is flat, and is a smooth function of $A$. For Dirac operators the relevant quantity is $\frac{1}{2}(\eta+h)$, where $h$ is the dimension of the kernel, and only its $(\bmod 1)$ reduction is smooth. The operator (1.27) is half the boundary operator associated to minus the signature operator. Thus its $\eta$-invariant takes real values. The $\eta$-invariant of this operator is minus the usual $\eta$-invariant, and we appropriately modify the formulae we quote from [APS]. The operator (1.27) is also the boundary operator associated to the self-duality operator, a fact we use below 12 We thank Ron Stern for discussions on this point. He points out that this argument makes clear the fact that this term in the asymptotic expansion (1.32) contributes a fourth root of unity, not an eighth root of unity
} 
This formula differs slightly from Witten's formula [W, (2.23)], and we explain the differences. First, the ratio of determinants in $[\mathrm{W},(2.8)]$ is the square root of the analytic torsion, whence the square root in (1.32). Also, [W,(2.16)] only holds $\left(\bmod \frac{1}{2}\right)$, which explains the appearance of the spectral flow (a fourth root of unity) in our formula. We have rewritten Witten's $\eta$-invariant contribution in terms of the spectral flow using (1.31). Witten's formula [W,(2.23)] has a prefactor which depends on the 2-framing of $X$. We claim that it vanishes for the canonical 2 -framing. The prefactor in question depends on a 2 -framing $\alpha$. It is the exponential of $2 \pi i$ times

$$
\frac{\eta(\theta)}{8}+\frac{\operatorname{CS}(\alpha)}{16}
$$

where $\operatorname{CS}(\alpha)$ is the Chern-Simons invariant of the Levi-Civity connection on $2 T(X)$ computed using the trivialization $\alpha^{13}$ Note that $\eta(\theta)$ is -3 times the usual $\eta$ invariant, since $\operatorname{dim} \mathfrak{s u}(2)=3$. Choose a 4-manifold $W$ such that $\partial W=X$ and $\sigma(W)=0$. Then by (1.3) the canonical 2-framing $\alpha$ extends to a 2 -framing over $W$. It follows that

$$
\operatorname{CS}(\alpha)=\frac{-1}{2 \pi^{2}} \int_{W} \operatorname{Tr}\left(\Omega^{2}\right)
$$

where $\Omega$ is the curvature of a Riemannian metric on $W$ which extends the given metric on $X$. This is the integral of the differential form which represents $t w i c e$ the first Pontrjagin class, the factor of 2 coming since we consider $2 T(X)$. On the other hand, the main theorem of [APS(i)] asserts

$$
\eta(\theta)=\frac{-1}{4 \pi^{2}} \int_{W} \operatorname{Tr}\left(\Omega^{2}\right)+\sigma(W) .
$$

It follows that (1.33) vanishes for the canonical framing. Finally, the factor of $1 / 2$ in (1.32) is due to the 2 elements in the center of $S U(2)$. These define global gauge transformations which act trivially on connections. The factor is a volume correction for this isotropy group. ${ }^{14}$

Formula (1.32) also holds if all flat connections are isolated (modulo gauge equivalence) and there is at least one irreducible connection; then the summation is over the irreducible connections. [The isolated reducible connections contribute lower order terms, as follows from (1.35).] Suppose now that all flat connections on $X$ are isolated and reducible. A prime example is $X=S^{3}$, where there is a single flat connection, the trivial connection $\theta$. Note $H_{X}^{0}(\theta) \cong \mathfrak{s u}(2)$ are the constant functions. In this case we have an explicit formula (1.13) for the partition function, from which we see

$$
Z_{S^{3}}(k) \sim \sqrt{2 \pi^{2}}(k+2)^{-3 / 2} .
$$

Of course, we could replace $k+2$ by $k$ in this formula. Notice that $I_{\theta}=S_{X}(\theta)=0$. The torsion $\tau_{S^{3}}(\theta)$ is not well-defined, due to the nontrivial cohomology. (It is well-

13 The embedding $S O(3) \hookrightarrow$ Spin(6) induces the diagonal embedding $\mathfrak{s D}(3) \hookrightarrow \mathfrak{s p}(6)$ on the Lie algebras. This explains the extra factor of 2 in the denominator of the second term. For more details about the classical Chern-Simons action, see [F1]. If the 2-framing $\alpha$ is induced by an ordinary framing, then (1.33) is 6 times the Adams e-invariant of that framing [APS(ii)]

${ }^{14}$ We thank Ed Witten for pointing this out to us 
defined only after choosing a volume form for the cohomology.) At this time we have no explanation for the factor $\sqrt{2 \pi^{2}}$. Still, we observe that the asymptotic formula has the additional factor

$$
(k+2)^{\left(\operatorname{dim} H_{X}^{1}(A)-\operatorname{dim} H_{X}^{0}(A)\right) / 2}
$$

which does not appear in (1.32). ${ }^{15}$ More generally, we speculate that

$$
\begin{aligned}
Z_{X}(k) & \sim \frac{1}{2} e^{-3 \pi i\left(1+b^{1}(X)\right) / 4} \\
& \times \sum_{i} \sqrt{\tau_{X}\left(A_{i}\right)} e^{-2 \pi i\left(I_{A_{i}} / 4+\operatorname{dim} H_{X}^{0}(A) / 8\right)} \\
& \times e^{2 \pi i S_{X}\left(A_{i}\right)(k+2)}(k+2)^{\left(\operatorname{dim} H_{X}^{1}(A)-\operatorname{dim} H_{X}^{0}(A)\right) / 2},
\end{aligned}
$$

if the flat connections $A_{i}$ are isolated modulo gauge. [Then $H_{X}^{1}\left(A_{i}\right)=0$, but we include it in the formula anyway.] At this time we do not know how to interpret the torsion $\tau_{X}\left(A_{i}\right)$ in (1.36). We will verify (1.36) for lens spaces and will note the behavior

$$
\left|Z_{X}(k)\right| \sim(k+2)_{A}^{\max _{A}\left(\operatorname{dim} H_{X}^{1}(A)-\operatorname{dim} H_{X}^{0}(A)\right) / 2}
$$

in some examples. This will include manifolds for which the flat connections are not isolated.

\section{Topology of 3-Manifolds}

In this section we describe the topological tools which we need for our computations. Our 3-manifolds are described by means of rational surgery, so we review this technique and the standard method of reducing to integer surgery. We state and prove a formula (2.4) for computing Atiyah's canonical 2-framing on any 3-manifold obtained by integer surgery on a link in $S^{3}$. We describe the Seifert manifolds which are the examples with which we test Witten's theory. We also provide computations of the invariants (signature, Chern-Simons invariant, Reidemeister torsion, etc.) which are relevant to our work. Finally, we describe a periodicity in Witten's invariants which is evident for certain families of 3-manifolds. (This periodicity has also been observed by Kirby and Melvin [KM2].)

2-Framings. Every oriented 3-manifold $X$ may be obtained by rational surgery on some link $L$ in $S^{3}$. This means that for each component $L_{i}$ of $L$, we remove a tubular neighborhood of $L_{i}$ (a solid torus) and glue it back in with a twist $M_{i}$. To specify $M_{i}$ we use a basis $\left\{m_{i}, l_{i}\right\}$ for $H_{1}$ of the boundary torus, where $l_{i}$ is the longitude running parallel to $L_{i}$ such that the linking number of $l_{i}$ with $L_{i}$ is zero, and $m_{i}$ is a small meridian of $L_{i}$, with a right-handed orientation relative to $l_{i}$. We now have

$$
M_{i}=\left(\begin{array}{ll}
p_{i} & r_{i} \\
q_{i} & s_{i}
\end{array}\right) \in S L(2 ; \mathbb{Z})
$$

15 Witten suggested this factor during an informal lecture in January, 1989. At that time he also showed some preliminary computations with the lens space $L(p, 1)$ similar to those in Sect. 4 
The diffeomorphism type of $X$ is specified by the link $L$ and the rational numbers $p_{i} / q_{i}$. (Here we allow $1 / 0$, corresponding to the identity matrix. If $q_{i}=0$, then $L_{i}$ may be deleted from the link.)

Fig. 2
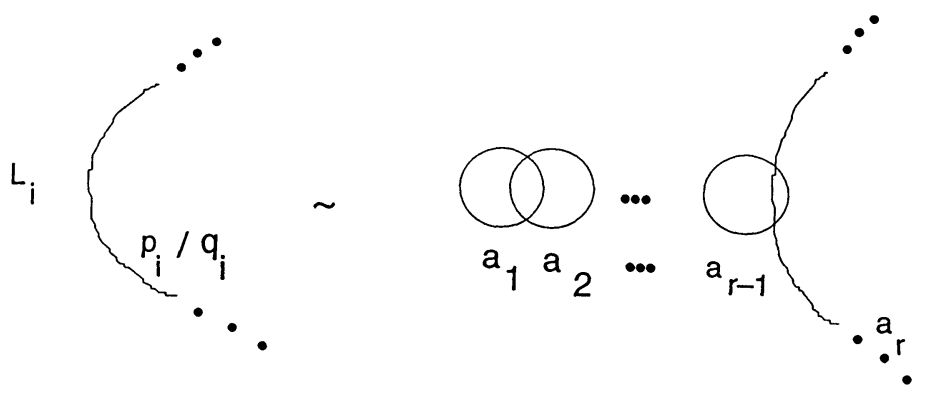

To specify $X$ as a 2-framed manifold, we need the entire matrix $M_{i}$, so we must establish a convention for recovering a matrix $M_{i}$ from $p_{i} / q_{i}$. We begin with integer surgery, or Kirby calculus [K] (all $q_{i}=1$ ), since in this case $X$ is exhibited explicitly as the boundary of a 4-manifold $W$, namely the handlebody obtained by gluing 2-handles to the 4-ball $B^{4}$ along the link $L C S^{3}=\partial B^{4}$ with normal framings specified by the integers $p_{i}$. In this case we choose

$$
M_{i}=\left(\begin{array}{rr}
p_{i} & -1 \\
1 & 0
\end{array}\right)=T^{p_{i}} S,
$$

where $T, S$ are the standard generators of $S L(2 ; \mathbb{Z})$ defined in (1.6). For any rational surgery, there is a method for reducing to an integer surgery [R]. Namely, for each $p_{i} / q_{i}$ choose a continued fraction expansion

$$
p_{i} / q_{i}=a_{r}-\frac{1}{a_{r-1}-\frac{1}{\ldots-\frac{1}{a_{1}}}}, \quad a_{i} \in \mathbb{Z},
$$

which we abbreviate $p_{i} / q_{i}=\left(a_{1}, \ldots, a_{r}\right)$. Then replace rational surgery on $L_{i}$ by integer surgery on a link, as indicated in Fig. 2. This does not change the diffeomorphism type of $X$, as may be seen by inductively applying the move shown in Fig. 3, which preserves diffeomorphism type. If the two gluing matrices on the left of Fig. 3 are

$$
M=\left(\begin{array}{ll}
p & r \\
q & s
\end{array}\right) \quad M^{\prime}=\left(\begin{array}{rr}
a & -1 \\
1 & 0
\end{array}\right),
$$

then the resulting matrix is

$$
M^{\prime} M=\left(\begin{array}{cc}
a p-q & a r-s \\
p & r
\end{array}\right) .
$$

Thus the natural way to specify a gluing matrix $M_{i}$ for $L_{i}$ with coefficient $p_{i} / q_{i}$ is to choose a continued fraction expansion $p_{i} / q_{i}=\left(a_{1}, \ldots, a_{r}\right)$ and apply the following formula:

$$
M_{i}=\left(\begin{array}{rr}
a_{r} & -1 \\
1 & 0
\end{array}\right)\left(\begin{array}{cr}
a_{r-1} & -1 \\
1 & 0
\end{array}\right) \ldots\left(\begin{array}{rr}
a_{1} & -1 \\
1 & 0
\end{array}\right)
$$


Our convention for dealing with rational surgeries is essentially a reduction to integer surgery. We emphasize that this depends on a choice of continued fraction expansion for each $p_{i} / q_{i}$. The matrices $M_{i}$ in (2.2) appear in the quantum field theory formulae (1.17) and (1.19) above.

If we cut a 2-framed 3-manifold $S$ along a torus and regule with a twist, the resulting manifold $X^{\prime}$ inherits a 2 -framing. To see this, note that since $\operatorname{Spin}(6)$ is 2 -connected, we may assume that the 2-framing restricts to the product (stable) 2 -framing on the torus $S^{1} \times S^{1}$. We may assume the twist is an element of $S L(2 ; \mathbb{Z})$, so that the new 2-framing induced by the twist differs from the old one at each point by an element of $S L(2 ; \mathbb{Z})$ which is constant over the torus. There is essentially a unique way to homotope this to the identity in $\operatorname{Spin}(6)$ through constants over the torus. This defines the 2 -framing on $X^{\prime}$. Since $\pi_{3}(\operatorname{Spin}(6)) \cong \mathbb{Z}$, there are different ways to do the initial straightening to a product 2-framing, but the resulting changes in the 2-framings on the pieces of $X$ will cancel in the final answer. (Note that this argument is specific to the torus. For higher genus surfaces, one must keep track of additional data.) Thus, by our convention (2.1), any link in $S^{3}$ with integer coefficients (and hence any link with rational coefficients and specified continued fraction expansions) determines a 2-framed 3-manifold via the canonical 2-framing on $S^{3}$.

Fig. 3
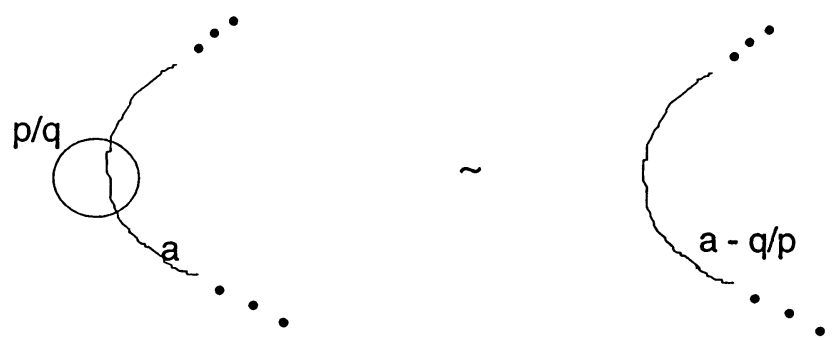

Let $L$ be a link in $S^{3}$ with integers $p_{1}, \ldots, p_{n}$ attached to the components. Let $X_{L}$ be the manifold obtained from this by surgery, and let $W_{L}$ be the corresponding 4-manifold with $\partial W_{L}=X_{L}$. The induced 2-framing on $X_{L}$ differs from the canonical 2 -framing of $X_{L}$ by an element of $\pi_{3}(\operatorname{Spin}(6)) \cong \mathbb{Z}$. Let $\varphi_{L}$ denote this integer. We determine the sign of $\varphi_{L}$ by the following convention: If we remove a solid torus from $X_{L}$ and glue it back in after a right-handed Dehn twist (i.e., the automorphism $T$ ) to obtain $X_{L}$ with a new 2-framing, the integer in question should increase. Note that by (1.7) this change in 2 -framing will multiply $Z_{X_{L}}$ by (1.4).

Theorem 2.3. We have

$$
\varphi_{L}=-3 \sigma\left(W_{L}\right)+\sum_{i=1}^{n} p_{i},
$$

where $\sigma$ denotes the signature of $W_{L}$ (or equivalently, the signature of the linking matrix for $L$ ).

We use this formula to correct (1.17) and (1.19) using (1.4).

Proof. We temporarily adopt a different convention about the gluing maps. If we replace each matrix (2.1) by

$$
M_{i}^{\prime}=\left(\begin{array}{cr}
p_{i} & -1 \\
1 & 0
\end{array}\right)\left(\begin{array}{cc}
1 & -p_{i} \\
0 & 1
\end{array}\right)=M_{i} T^{-} p_{i}
$$


then the diffeomorphism type of $X_{L}$ does not change, but the new induced 2 -framing differs from the canonical framing by a different integer $\psi_{L}$. In fact, each right multiplication by $T$ changes the 2-framing by one unit. (We verify this by direct computation in Appendix B.) Thus, we have

$$
\psi_{L}=\varphi_{L}=\sum p_{i}
$$

by the sign convention specified above. Note that if we let $l_{i}^{\prime}=l_{i}+p_{i} m_{i}$ be the longitude determined by the framing $p_{i}$, then $M_{i}^{\prime}$ maps $m_{i}$ to $l_{i}^{\prime}$ and $l_{i}^{\prime}$ to $-m_{i}$. From this description it is easy to see that $\psi_{L}$ does not change under isotopy or handle slides.

It now suffices to show that $\psi_{L}=-3 \sigma\left(W_{L}\right)$. First, we show that blowing up a \pm 1 changes $\psi_{L}$ by $\mp 3$. If $L_{+}$denotes the unknot with coefficient +1 , then the gluing map determining $\psi_{L_{+}}$is

$$
M^{\prime}=\left(\begin{array}{rr}
1 & -1 \\
1 & 0
\end{array}\right) T^{-1}
$$

The manifold $X_{L_{+}}$is diffeomorphic to $S^{3}$, as can be seen by putting a left twist in the solid torus forming the knot complement, to obtain $1 / 0$ surgery on the unknot. This action changes $M^{\prime}$ by left multiplication by

$$
\left(\begin{array}{rr}
1 & 0 \\
-1 & 1
\end{array}\right)=S T S^{-1}
$$

yielding the matrix $T^{-2}$. Thus, $X_{L_{+}}$is made from $S^{3}$ by putting two left twists $\left(T^{-2}\right)$ in the tubular neighborhood of an unknot, one left twist in the complement, and then gluing the pieces back together by the identity map. The exhibits $X_{L_{+}}$as $S^{3}$ with 2-framing differing from the canonical one by -3 twists, so $\psi_{L_{+}}=-3$. Similarly, if $L_{-}$is the unknot with coefficient -1 , we have $\psi_{L_{-}}=3$. These arguments are not affected by surgeries far away from $L_{ \pm}$. Since blowing up a \pm 1 is the same as adding a copy of $L_{ \pm}$far away from a given link and then sliding handles, we have that blowing up \pm 1 adds $\mp 3$ to $\psi_{L}$.

If $L_{*}$ is obtained from $L$ by adding $n$ to one surgery coefficient (thereby changing the diffeomorphism type of $X_{L}$ ), then

$$
\psi_{L_{*}}-\psi_{L}=-3 \sigma\left(W_{L *}\right)+3 \sigma\left(W_{L}\right) .
$$

To verify this, let $\lambda$ denote $\frac{1}{2} p_{1}\left(2 T\left(W_{L}\right), \alpha_{L}\right)$, where $\alpha_{L}$ is the 2 -framing inherited by $X_{L}$, and let $\lambda_{*}=\frac{1}{2} p_{1}\left(2 T\left(W_{L_{*}}\right), \alpha_{L_{*}}\right)$. Then by Atiyah's formula for the canonical framing (1.3),

$$
\psi_{L}= \pm\left(3 \sigma\left(W_{L}\right)=\lambda\right),
$$

and similarly for $L_{*}$. The manifold $W_{L *}$ is obtained from $W_{L *}$ by cutting along a solid torus, adding $n$ twists, and regluing. This changes the relative framing by $n$ units. However, the longitude used to compute $\psi$ also changes by $n$ twists. These twists must calcel, since $\psi_{L_{+}}-\psi_{L_{-}}=-6$. Thus, $\lambda=\lambda_{*}$ and

$$
\psi_{L *}-\psi_{L}= \pm\left(3 \sigma\left(W_{L *}\right)-3 \sigma\left(W_{L}\right)\right) .
$$

The ambiguous sign is - , by our computation for $L_{ \pm}$.

It is now easy to see that $\psi_{L}=-3 \sigma\left(W_{L}\right)$ for $L$ an unlink with any integer coefficients. To complete the proof, it suffices to show that if $L$ is any link with integer coefficients and if $L^{\prime}$ differs from $L$ by a single crossing change (and some 
surgery coefficients), then

$$
\psi_{L^{\prime}}-\psi_{L}=-3 \sigma\left(W_{L^{\prime}}\right)+3 \sigma\left(W_{L}\right)
$$

Figure 4 demonstrates this. In this picture (a) and (c) have the same values of $\psi$ and $\sigma$. When we change to (d), then $\psi$ changes by -3 times the difference in signatures. We move to (e) by the usual procedure of separating the Hopf link from the rest and then canceling it by blowing up and down; this preserves $\psi$ and $\sigma$.

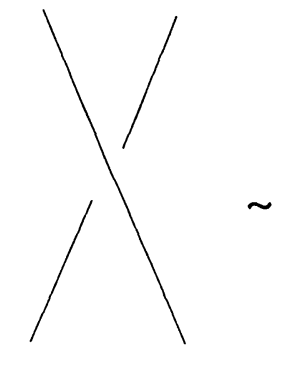

$\mathbf{a}$

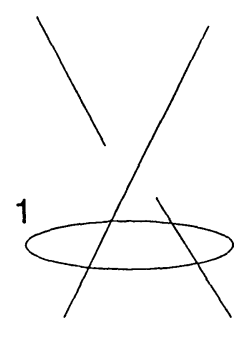

b

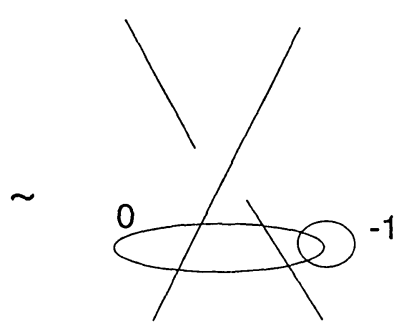

c
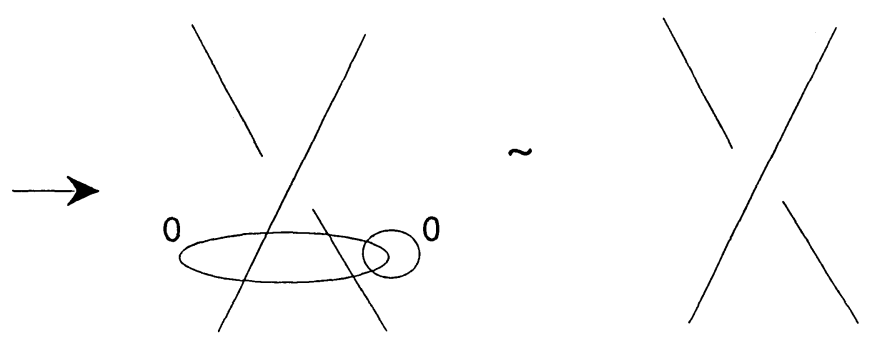

Fig. 4

d

e

To compute Witten's invariant with respect to the canonical 2-framing we must apply Theorem 2.3. In particular, we must compute the signature $\sigma\left(W_{L}\right)$ for our examples. We begin with the lens space $L(p, q)$ with $p>|q| \geqq 1$ (1.18) which is more conveniently described as $-p / q$ surgery on the unknot in $S^{3}$. (See Fig. 3.) Choose a continued fraction expansion $-p / q=\left(a_{1}, \ldots, a_{r}\right)$ with $\left|a_{i}\right| \geqq 2$ (for example, by repeatedly decomposing as an integer of absolute value $\geqq 2$ plus remainder). Then by Fig. 2 the lens space $L(p, q)$ is described by the integer surgery diagram shown in Fig. 5. The linking matrix $\left(a_{i j}\right)$ for this surgery diagram has diagonal entries $a_{i i}=a_{i}$, and has $a_{i, i \pm 1}=1$. All other entries are zero. Since all $\left|a_{i}\right| \geqq 2$, diagonalization shows that the signature of $\left(a_{i j}\right)$ is

$$
\sigma\left(W_{L}\right)=\sum_{i} \operatorname{sign}\left(a_{i}\right)
$$

where $\operatorname{sign}(a)=1,0,-1$ according as $a>0, a=0$, or $a<0$.

Next, we consider a general rational surgery diagram $L_{0}$. As in Fig. 2, we obtain an integer surgery diagram $L$ by continued fraction expansions $\left(a_{1}, \ldots, a_{r}\right)$ of each nonintegral coefficient of $L_{0}$. We may assume $\left|a_{i}\right| \geqq 2$ for each $i \leqq r-1$. Let 


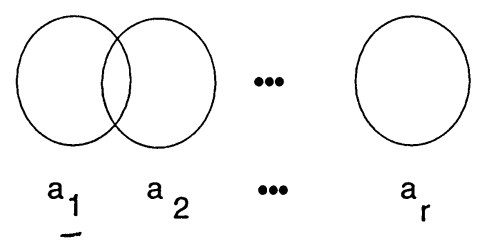

$\left\{b_{j}: 1 \leqq j \leqq N\right\}$ denote the family of integers obtained by deleting the last entry of each continued fraction and combining the remaining numbers into a single collection. Let $A$ denote the linking matrix of $L_{0}$; the diagonal entries are the (rational) surgery coefficients of $L_{0}$ and the off-diagonal entries are linking numbers. Then we have

$$
\sigma\left(W_{L}\right)=\sigma(A)+\sum_{j=1}^{N} \operatorname{sign}\left(b_{j}\right) .
$$

This may be seen by reducing the matrix for $L$. (For each nonintegral surgery coefficient of $L_{0}$, apply a diagonalization procedure similar to the one which works for lens spaces.)

We apply the preceding formula to the Seifert manifold $X\left(p_{1} / q_{1}, \ldots, p_{n} / q_{n}\right)$ (1.15). Recall that this manifold is obtained from $S^{2} \times S^{1}$ by rational surgery on $n$ circles $p t \times S^{1}$. Surgery on another such circle changes $S^{2} \times S^{1}$ into $S^{3}$, resulting in the rational surgery diagram for $X\left(p_{1} / q_{1}, \ldots, p_{n} / q_{n}\right)$ shown in Fig. 6. [Note that if we compute Witten's invariant directly from this picture, we recover (1.17).] If we choose continued fraction expansions to obtain an integer surgery diagram $L$, Eq. (2.6) immediately yields

$$
\sigma\left(W_{L}\right)=-\operatorname{sign}\left(\sum_{i=1}^{n} q_{i} / p_{i}\right)+\sum_{i=1}^{n} \operatorname{sign}\left(p_{i} / q_{i}\right)+\sum_{j=1}^{N} \operatorname{sign}\left(b_{j}\right) .
$$

Here, the first term is taken to be zero if any $p_{i}=0$.

Fig. 6

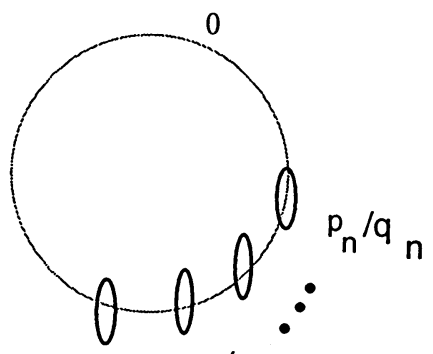

$$
p_{1} / q_{1} \quad p_{2}^{\prime} q_{2}
$$

Seifert Manifolds. We now list some general facts about lens spaces and other Seifert manifolds. The fundamental group of the lens space $L(p, q)$ is $\mathbb{Z} / p \mathbb{Z}$. Two lens spaces $L(p, q)$ and $L\left(p, q^{\prime}\right)$ are diffeomorphic if and only if

$$
q \equiv \pm q^{\prime}(\bmod p) \quad \text { or } q q^{\prime} \equiv \pm 1(\bmod p)
$$

A diffeomorphism preserves orientation if and only if the relevant sign is + . Therefore, the lens space $L(p, q)$ has an orientation reversing self-diffeomorphism if 
and only if

$$
q^{2} \equiv-1(\bmod p)
$$

For example, $L(5,2) \approx L(5,3) \approx-L(5,2) \approx-L(5,3)$ as oriented manifolds. Notice that $L(0,1)=S^{2} \times S^{1}$ and $L(1,0) \approx S^{3}$. By the above relations all other oriented diffeomorphism types of lens spaces are realized by $p \geqq 2$ and $1 \leqq q \leqq p-1$. An important feature of lens spaces is that many homotopy types contain more than one diffeomorphism type. In fact, there is a homotopy equivalence between $L(p, q)$ and $L\left(p, q^{\prime}\right)$ if and only if

$$
q^{\prime} \equiv \pm n^{2} q(\bmod p)
$$

for some integer $n$, and the map preserves orientation if and only if the sign is + . For example, the lens spaces $L(7,1)$ and $L(7,2)$ have the same oriented homotopy type, but they are not diffeomorphic.

Consider, once again, the Seifert manifold $X\left(p_{1} / q_{1}, \ldots, p_{n} / q_{n}\right)$. We allow $p_{i}=0$, even though the resulting manifold is not, strictly speaking, a Seifert fibered space, but rather is a connected sum of lens spaces:

$$
X\left(p_{1} / q_{1}, \ldots, p_{n} / q_{n}, 0\right) \approx \# L\left(-p_{i}, q_{i}\right),
$$

as is easily derived from Fig. 6 . We will be primarily interested in the case $n=3$, although all of our work holds for the general case. The order of the first homology group of $X\left(p_{1} / q_{1}, p_{2} / q_{2}, p_{3} / q_{3}\right)$ is the absolute value of

$$
p_{1} p_{2} q_{3}+p_{1} q_{2} p_{3}+q_{1} p_{2} p_{3} \text {. }
$$

(Here zero is understood to indicate infinite order.) In particular, we can choose $q_{1}, q_{2}$, and $q_{3}$ to obtain a homology sphere if and only if $p_{1}, p_{2}$, and $p_{3}$ are pairwise relatively prime. In this case the manifold is independent of $q_{1}, q_{2}, q_{3}$, and it is called the Brieskorn homology sphere $\Sigma\left(p_{1}, p_{2}, p_{3}\right)$. [Without loss of generality we can take all $p_{i}>0$. We choose $q_{1}, q_{2}, q_{3}$ so that (2.9) is positive. This has the effect of orienting $\Sigma\left(p_{1}, p_{2}, p_{3}\right)$ as the link of the algebraic singularity $z_{1}^{p_{1}}+z_{2}^{p_{2}}+z_{3}^{p_{3}}=0$.] We will illustrate the Witten invariant for the family

$$
\Sigma(2,3,6 n \pm 1)=X(2 / \pm 1,3 / \mp 1,(6 n \pm 1) / \mp n) \text {. }
$$

Now two of these are diffeomorphic. Note that $\Sigma(2,3,1) \approx S^{3}$. The manifold $\Sigma(2,3,5)$ is the famous Poincare homology sphere, and for each nonnegative integer $n$ the homology sphere $\Sigma(2,3,6 n \pm 1)$ may be described as $-1 / n$ surgery on the trefoil knot, where the knot is right or left handed depending on whether the sign is + or - . More generally, rational surgery on the left-handed trefoil knot with any coefficient $p / q$ is realized by

$$
X(-2 / 1,3 / 1,(p+6 q) / q) \text {. }
$$

We will consider the family $X_{\text {tref }}(p)$ of integer surgeries on the left trefoil with coefficient $p \in \mathbb{Z}$. We have $H_{1}\left(X_{\text {tref }}(p)\right) \cong \mathbb{Z} /|p| \mathbb{Z}$. The manifold $X_{\text {tref }}(0)$ is of particular interest since its homology is infinite cyclic, and because it is a torus bundle over $S^{1}$. The monodromy matrix of this torus bundle is

where

$$
N=\left(\begin{array}{rr}
0 & -1 \\
1 & 1
\end{array}\right),
$$

$$
X_{\text {tref }}(0) \approx T^{2} \times I /(x, 0) \sim(N x, 1) .
$$


Using the torus bundle structure of $X_{\text {tref }}(0)$, we may compute its Witten invariants directly by (1.24), but this formula assumes a particular (non-canonical) 2 -framing. In fact, $X_{\text {tref }}(0)$ is obtained from $T^{2} \times S^{1}$ by cutting along $T^{2} \times p t$ and regluing after twisting by $N$. The 2-framing required by (1.24) is the one inherited from the canonical 2-framing on $T^{2} \times S^{1}$ by the regluing. (This is well-defined by the discussion preceding Theorem 2.3.) To compare this 2-framing with the canonical one, decompose $N$ as a product of two Dehn twists about two orthogonal axes $C_{1}$ and $C_{2}$ in $T^{2}$ :

$$
N=\left(\begin{array}{rr}
1 & -1 \\
0 & 1
\end{array}\right)\left(\begin{array}{ll}
1 & 0 \\
1 & 1
\end{array}\right)=\left(T^{-1}\right)\left(S T^{-1} S^{-1}\right)
$$

We may assume that the Dehn twists have support in small neighborhoods of $C_{1}$ and $C_{2}$, respectively. Now if we think of $X_{\text {tref }}(0)$ as made from $T^{2} \times S^{1}$ by cutting and regluing by these twists near $C_{1} \times 1$ and $C_{2} \times-1$, we may reinterpret the procedure as surgery on the two circles $C_{1} \times 1$ and $C_{2} \times-1$ in $T^{2} \times S^{1}$. The matrix of each surgery will be

$$
M=\left(\begin{array}{ll}
1 & 0 \\
1 & 1
\end{array}\right) .
$$

(The basis $\left\{m_{i}, l_{i}\right\}$ is chosen so that $l_{i}$ respects the product structure of $T^{2} \times S^{1}$.) We examine this in the standard Kirby calculus picture: $T^{2} \times S^{1}$ is 0 -surgery on the Borromean rings, and $C_{1} \times 1$ and $C_{2} \times-1$ are meridians of two of the link components. The matrix $M$ indicates that we perform +1 surgery on each of these to obtain $X_{\text {tref }}(0)$ as a surgery on a link $L C S^{3}$. To understand the inherited 2 -framing using Theorem 2.3, we must replace the matrix $M$ with our standard matrix (2.1)

$$
\left(\begin{array}{rr}
1 & -1 \\
1 & 0
\end{array}\right)=M T^{-1}
$$

This changes our 2-framing by two applications of $T^{-1}$ (one for each surgery). The new 2-framing differs from the canonical one by $\varphi_{L}=2$, by (2.4). Thus, we must apply 4 left twists $\left(T^{-1}\right)$ to get from the original bundle 2-framing to the canonical one.

The Seifert space formalism also allows us to study torus knots. As an example,

$$
X(-2 / 1,3 / 1) \approx S^{3} \text {. }
$$

Let $C \subset X(-2 / 1,3 / 1)$ be a third meridian to the 0 -framed circle in Fig. 6 [so that $p / q$ surgery on $C$ would result in $X(-2 / 1,3 / 1, p / q)]$. Then by elementary Kirby calculus, the circle $C$ corresponds to the left-handed trefoil knot in $S^{3}$. The 0 -framing on $C$ as it appears in Fig. 6 corresponds to the -6 framing on the lefthanded trefoil. [This justifies (2.11).] Thus, we may compute the corresponding Witten invariant $Z_{\left(S^{3} \text {; tref }\right)}$ from Fig. 6, as long as we remember to compensate for the normal framing.

$M u$ Invariants. In Sect. 4 we need to compute $m u$-invariants. Given a 3-manifold $X$ with a spin structure $\Theta$, the mu invariant of $X$ with respect to $\Theta$ is the signature (reduced modulo 16) of any spin 4-manifold whose boundary is $(X, \Theta)$. Any two spin structures on $X$ differ by an element of $H^{1}(X ; \mathbb{Z} / 2 \mathbb{Z})$. First we consider the lens space $L(p, q)$. Assume $p>|q|>0$, and expand $-p / q$ as a continued fraction 
$\left(a_{1}, \ldots, a_{r}\right)$ with all $\left|a_{i}\right| \geqq 2$ and all $a_{i}$ even for $i \neq 1$ (for example, by repeatedly decomposing as an even integer plus a remainder with absolute value less than one). Consider the integer surgery diagram for $L(p, q)$ shown in Fig. 5. First, suppose $a_{1}$ is even. Then the 4-manifold $W_{L}$ in Fig. 5 is spin. (In general, for any link $L$ with integer coefficients, $W_{L}$ is spin if and only if all coefficients are even.) Thus, the mu invariant of $L(p, q)$ for one choice of spin structure is given by (2.5):

$$
\sigma\left(W_{L}\right)=\sum_{i} \operatorname{sign}\left(a_{i}\right) .
$$

If $p$ is odd, then $H^{1}(L(p, q) ; \mathbb{Z} / 2 \mathbb{Z})=0$ and this is the only spin structure. Otherwise, there are exactly two spin structures. The mu invariant of the other structure may be obtained as in [Ka]. By an easy induction on $r$, we see that $r$ is odd if $p$ and all $a_{i}$ are even. Slide the first link component $L_{1}$ over all other components $L_{i}$ with $i$ odd to obtain an unknot $K$ with framing $a=\sum_{i \text { odd }} a_{i}$. If $a \geqq 2$ blow up -1 -framed meridians to drop the framing of $K$ to 1 ; then blow down $K$. The result is an even 4-manifold realizing the other spin structure with signature

$$
\sum_{i} \operatorname{sign}\left(a_{i}\right) \sum_{i \equiv r(\bmod 2)} a_{i} .
$$

If $a=0$ or $a \leqq-2$, a similar argument also yields (2.15). The mod 16 residue of (2.15) is the second mu invariant of $L(p, q)$ for $p$ even. In the one remaining case, where $a_{1}$ is odd, $p$ must also be odd, so that there is a unique spin structure. By the same argument, sliding $L_{1}$ (if $r$ is odd) or $L_{2}$ (if $r$ is even) over all other $L_{i}, i \equiv r(\bmod 2)$, we obtain (2.15) as our mu invariant.

Similar computations work for other manifolds. Any homology sphere has a unique spin structure. For $\Sigma(2,3,6 n \pm 1)$ it is well-known that the mu invariant is

$$
\mu(\Sigma(2,3,6 n \pm 1)) \equiv 8 n(\bmod 16) .
$$

The manifold $X_{\text {tref }}(p)$ has one or two spin structures according as $p$ is odd or even. For any $p \geqq-5$ we expand $X_{\text {tref }}(p)$ as in Fig. 7, by Kirby calculus. One mu invariant will be the signature of the 4-manifold on the right of Fig. 7, which is

$$
\mu\left(X_{\text {tref }}(p)\right)=-p-8+\operatorname{sign}(p) .
$$

(Similar calculation involving +2 's show that this formula is valid for all $p$.) If $p$ is even, the other mu invariant is $\operatorname{sign}(p)$, the signature of the spin manifold $W_{L}$ for $L$ the trefoil with coefficient $p$.

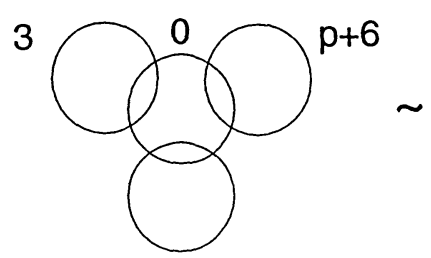

Fig. 7

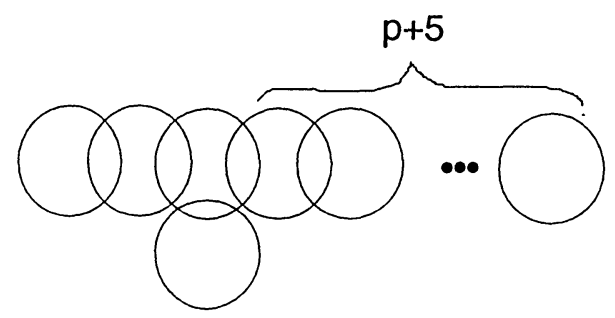

all -2

Chern-Simons Invariants and Spectral Flow. We consider lens spaces. Since $\pi_{1}(L(p, q)) \cong \mathbb{Z} / p \mathbb{Z}$ is cyclic, each of the representations $\pi_{1}(L(p, q)) \rightarrow S U(2)$ is 
reducible. Let $\zeta$ be a generator of the fundamental group, and set $\omega=e^{2 \pi i / p}$. The trivial connection $\theta$ and (if $p$ is even) the representation $\zeta \mapsto-1$ have $\operatorname{dim} H^{0}=3$, while the other equivalence classes of representations

$$
A_{n}: \zeta \mapsto\left(\begin{array}{cc}
\omega^{n} & \\
& \omega^{-n}
\end{array}\right), \quad 0<n<\frac{p}{2},
$$

have $\operatorname{dim} H^{0}=1$. The first cohomology vanishes for all representations. According to (1.36) it is only the representations (2.18) which contribute to the leading term of the large $k$ asymptotics of $Z_{X}(k)$. The $\varrho$-invariant (1.29) of the representation $\zeta \mapsto \omega^{n}$ into $U(1)$ can be computed from the formulae in [APS(ii), Sect. 2]. ${ }^{16}$ We find the value

$$
\frac{1}{p} \sum_{k=1}^{p-1} \cot \left(\frac{\pi k}{p}\right) \cot \left(\frac{\pi q k}{p}\right)\left(\omega^{n k}-1\right) .
$$

However, we must compute this invariant in the adjoint of the representation (2.18), and so

$$
\varrho\left(A_{n}\right)=\frac{2}{p} \sum_{k=1}^{p-1} \cot \left(\frac{\pi k}{p}\right) \cot \left(\frac{\pi q k}{p}\right)\left(\omega^{2 n k}-1\right) .
$$

The Chern-Simons invariant is computed (by a different method) in [KK] to be

$$
S_{X}\left(A_{n}\right)=\frac{q^{*} n^{2}}{p}(\bmod 1)
$$

where $q^{*} q \equiv 1(\bmod p)$. [Presumably this could be derived directly from (2.19) using (1.29).] Hence from (1.31) the spectral flow is

$$
I_{A_{n}} \equiv \frac{8 q^{*} n^{2}}{p}-\frac{1}{p} \sum_{k=1}^{p-1} \cot \left(\frac{\pi k}{p}\right) \cot \left(\frac{\pi q k}{p}\right)\left(\omega^{2 n k}-1\right)-2 \quad(\bmod 8) .
$$

Let $X=\Sigma\left(p_{1}, p_{2}, p_{3}\right)$ be a Brieskorn homology sphere. Recall from the discussion following (2.9) that we choose the other Seifert invariants $q_{1}, q_{2}, q_{3}$ so that

$$
p_{1} p_{2} q_{3}+p_{1} q_{2} p_{3}+q_{1} p_{2} p_{3}=1 .
$$

Then the fundamental group has a presentation

$$
\left.\pi_{1}(X) \cong\left\langle x_{1}, x_{2}, x_{3}, h\right| h \text { central, } x_{i}^{p_{i}} h^{q_{i}}=1, x_{1} x_{2} x_{3}=1\right\rangle .
$$

Now there is a finite number of irreducible representations $\pi_{1}(X) \rightarrow S U(2)$ up to conjugacy; they are described in [FS, Sect. 2]. The conjugacy class is determined by integers $l_{i}$ with $0<l_{i}<p_{i}$ such that the matrix representing $x_{i}$ is conjugate to

$$
\left(\begin{array}{cc}
e^{\pi i i_{i} / p_{i}} & \\
& e^{-\pi i i_{i} / p_{i}}
\end{array}\right) .
$$

Fix a triple $\left\langle l_{1}, l_{2}, l_{3}\right\rangle$ of integers describing such a representation, and let $A$ denote the associated flat connection. Then the formula for the Chern-Simons invariant is

${ }^{16}$ Recall from (1.27) that we must change the sign of the formulae in [APS] 
given in $[\mathrm{FS}]$ and $[\mathrm{KK}]$ as $^{17}$

$$
S_{X}(A) \equiv \frac{-e^{2}}{4 p}(\bmod 1),
$$

where $p=p_{1} p_{2} p_{3}$ and $e=l_{1} p_{2} p_{3}+p_{1} l_{2} p_{3}+p_{1} p_{2} l_{3}$. For the spectral flow we quote [FS, Sect. 3]:

$$
I_{A} \equiv-3-\left[\frac{2 e^{2}}{p}+\sum_{i=1}^{3} \frac{2}{p_{i}} \sum_{k=1}^{p_{i}-1} \cot \left(\frac{\pi p k}{p_{i}^{2}}\right) \cot \left(\frac{\pi k}{p_{i}}\right) \sin ^{2}\left(\frac{\pi e k}{p_{i}}\right)\right](\bmod 8) .
$$

Reidemeister Torsion. The Reidemeister torsion of lens spaces was computed in a classic paper of Franz [Fr] (cf. [RS2, Sect.1]), who used this invariant to distinguish the various $L(p, q)$. In fact, some of his results were given in (2.8). Consider the one dimensional representation $\zeta \mapsto \omega^{n}$ of $\pi_{1}(L(p, q))$ for $0<n<\frac{p}{2}$, where $\omega=e^{2 \pi i / p}$. Then Franz' formula for the torsion, suitably normalized, is

$$
\left|\omega^{n}-1\right|\left|\omega^{q^{*} n}-1\right|,
$$

where $q^{*} q \equiv 1(\bmod p)$. We need the torsion of the connection $(2.18)$ in the adjoint representation, which is the $\operatorname{sum} \zeta \mapsto \omega^{2 n} \oplus 1 \oplus \omega^{-2 n}$ of one dimensional representations. Leaving aside the trivial representation, we compute the torsion

$$
\left|\omega^{2 n}-1\right|\left|\omega^{2 q^{* n}}-1\right|\left|\omega^{-2 n}-1\right|\left|\omega^{-2 q^{* n}}-1\right|=2^{4} \sin ^{2}\left(\frac{2 \pi n}{p}\right) \sin ^{2}\left(\frac{2 \pi q^{*} n}{p}\right)
$$

of the remaining two dimensional representation. Now the trivial representation has nonzero homology, so we must choose a basis for the homology to define the torsion. With an obvious choice for this basis, an easy computation [RS, Sect. 1] shows that the torsion of the trivial representation is $1 / p$. Hence

$$
\sqrt{\tau_{L(p, q)}\left(A_{n}\right)}=\frac{4}{\sqrt{p}}\left|\sin \left(\frac{2 \pi n}{p}\right) \sin \left(\frac{2 \pi q^{*} n}{p}\right)\right| .
$$

The Reidemeister torsion for the Brieskorn sphere $X=\Sigma\left(p_{1}, p_{2}, p_{3}\right)$ is computed in [F2, Sect. 2]. [There Franz' formula (2.24) is also rederived.] Let the flat $S U(2)$ connection $A$ be described by the triple of integers $\left\langle l_{1}, l_{2}, l_{3}\right\rangle$. Then the square root of the torsion in the adjoint representation is

$$
\sqrt{\tau_{X}(A)}=\frac{8}{\sqrt{p}} \prod_{i=1}^{3}\left|\sin \left(\frac{\pi q_{i}^{*} l_{i}}{p_{i}}\right)\right|,
$$

where $p=p_{1} p_{2} p_{3}$ and $q_{i}^{*} q_{i} \equiv 1\left(\bmod p_{i}\right)$.

A Periodicity Phenomenon. Witten invariants display a certain periodicity under changing surgery coefficients, with period depending on the level $k$. Suppose $L$ is a link with integer surgery coefficients, and let $L^{\prime}$ be obtained from $L$ by adding $n$ twists to the coefficient of one component $L_{1}$. Assume that $\sigma\left(W_{L^{\prime}}\right)=\sigma\left(W_{L}\right)$. (If $L$ is a

17 This is minus the result in [KK] and $-1 / 4$ times the formula in [FS] for the Chern-Simons invariant of the adjoint connection. The discrepancy with $[\mathrm{KK}]$ arises due to the sign convention in [FS] that $p_{1}$ of a real vector bundle is minus $c_{2}$ of the complexified bundle 
knot, this holds provided the sign of the surgery coefficient is not changed.) Then the Witten invariant of $X_{L}$ at level $k$ with the canonical 2-framing has the form

$$
Z_{X_{L}}=\langle M(\mathbf{1}), \psi\rangle
$$

where $\langle\alpha, \psi\rangle=\mathrm{Z}_{\left(X ;\left(L_{1}, \boldsymbol{\alpha}\right)\right)}$ is the invariant of the pair $\left(X ; L_{1}\right)$ obtained by labeling $L_{1}$ with label $\alpha$ and performing surgery on the other link components. Then the invariant of $X_{L^{\prime}}$ is

$$
Z_{X_{L^{\prime}}}=\left\langle\widetilde{T}^{n} M \tilde{T}^{-n}(\mathbf{1}), \psi\right\rangle,
$$

where the factor $\widetilde{T}^{-n}$ is added to correct the 2-framing using (2.4). [If $\sigma\left(W_{L^{\prime}}\right)$ $\neq \sigma\left(W_{t}\right)$, then formula (2.27) is off by an easily computed root of unity.] By (1.7) the term $\widetilde{T}^{-n}$ has the effect of scalar multiplication by $e^{2 \pi i n c / 24}$, which cancels the terms involving $c$ in the $\widetilde{T}^{n}$ factor. Thus, we change $Z_{X_{L}}$ to $Z_{X_{L}}$ by multiplying some terms by roots of unity $e^{2 \pi i n h_{\alpha}}$. If $n$ is a multiple of $4(k+2)$, then these roots are all equal to 1 , by (1.9), whence $Z_{X_{L^{\prime}}}=Z_{X_{L}}{ }^{18}$

The periodicity applies to the family of homology spheres $X_{q}$ obtained by $1 / q$ surgery on a fixed knot $K$ in $S^{3}$, since $X_{q}$ is obtained by surgery on the link $L_{q}$ given by $K$ with coefficient 0 and a meridian to $K$ with coefficient $-q$. (See Fig. 3.) We have $\sigma\left(W_{L_{q}}\right)=0$ independent of $q$. Thus,

$$
Z_{X_{q}}=Z_{X_{q+n}}
$$

where $n$ is any multiple of $4(k+2)$. Our data for the left trefoil reveals a smaller period $k+2$. This $(k+2)$-periodicity has been shown to hold for arbitrary knots in $S^{3}$ by Kirby and Melvin [KM2].

\section{Method}

The main calculations of the Witten invariant were coded using the standard C programming language. Many of the routines were first developed in Mathematica [Wol]. We also used Mathematica to carry out smaller computations. The programs were developed on NeXT computers and run on both NeXTs and a Sun 4/370 SPARCserver.

Our code was tested in several ways. First, many of the routines were run in both $\mathrm{C}$ and Mathematica. Also, we were able to test our routines for lens space calculations against the routines for Seifert spaces, since by (1.19) the lens space $L(p, q)$ is the Seifert space $X(q / p)$. Other coincidences such as

$$
\begin{gathered}
L(5,4)=X(-2 / 1,3 / 1,1 / 1), \\
L(7,2)=X(-2 / 1,3 / 1,-1 / 1)
\end{gathered}
$$

provided more tests. In addition, the connected sum $L\left(p_{1}, q_{1}\right) \# L\left(p_{2}, q_{2}\right)$ is the manifold $X\left(0 / 1,-p_{1} / q_{1},-p_{2} / q_{2}\right)$. So we further tested our routines using (1.11). Finally, after reading Sect. 4 we hope the reader will not imagine that computations so consistent with theory could come from defective programs.

${ }^{18}$ A similar periodicity (with different periods) occurs for other Lie groups 


\section{Results}

We are ready to present and analyze our computations. The computed values for the Witten invariant obey the predictions of Quantum Field Theory and of Topology. The reader should notice the following general features. First, if $Z_{X} \neq Z_{X^{\prime}}$, then $X$ is not (orientation preserving) diffeomorphic to $X^{\prime}$. This is consistent with Witten's assertion that the path integral is a topological invariant. Furthermore, we observe that the invariant conjugates under orientation reversal, consistent with (1.10). For manifolds with orientation reversing selfdiffeomorphisms, the invariant is real. Finally, this invariant turns out to be quite effective. This is most striking in the case of lens spaces, which historically were first distinguished by the Reidemeister torsion. Here it is crucial that we use the canonical 2-framing, and not just consider the invariant up to certain roots of unity. For most values of the level $k$ the invariant distinguishes many different lens spaces, even those which have the same oriented homotopy type. Perhaps our best results come when we examine the invariant for large values of $k$. There we verify (numerically) the predicted asymptotic formula.

Tabulations. Lens spaces. The values of the invariant for lens spaces can be computed by combining (1.19) and (2.2), though we actually use the equivalent procedure indicated above. We expand $L(p, q)$ as in Fig. 5 , compute $(\widetilde{S} \tilde{M})_{1}^{1}$ with $M$ as in (2.2), and correct the framing with (2.4).

The Witten invariants for the lens spaces $L(5, q)$ are displayed in Table 1 . These numbers are consistent with the diffeomorphisms

$$
L(5,1) \approx-L(5,4) \text { and } L(5,2) \approx L(5,3) \approx-L(5,2) \approx-L(5,3)
$$

which follow from (2.8). Notice that $k=2$ already reflects the fact that no further relations are possible, as do most other values of $k$. The invariant is real for $L(5,2)$ and $L(5,3)$, since these manifolds have orientation reversing self-diffeomorphisms. We display explicit values for some large $k$ in anticipation of the discussion below.

The lens spaces $L(7,1)$ and $L(7,2)$ are orientation preserving homotopy equivalent, but not diffeomorphic. Table 2 demonstrates the ability of the Witten invariant to distinguish these spaces. Notice that the Witten invariants differ except for $k=1$ and $k=4$. In fact, for $k \leqq 125$ these are the only values of $k$ for which the Witten invariant does not distinguish the spaces. The third column of Table 2 illustrates the importance of the canonical 2-framings; we check those values of $k$ for which the invariants of $L(7,1)$ and $L(7,2)$ are different, and not simply by a power of $e^{-2 \pi i c / 24}$, where $c$ is the central charge (1.5). In other words, only for the marked values of $k$ does the invariant distinguish the spaces without using the canonical 2-framings. There are very few such values of $k$.

The lens spaces $L(25,4)$ and $L(25,9)$ are also homotopy equivalent, but the Witten invariant behaves somewhat differently. ${ }^{19}$ We computed the invariants for $1 \leqq k \leqq 200$; the values for $1 \leqq k \leqq 20$ are shown in Table 3 . Here we observe that the invariants agree for $k \neq 3(\bmod 5)$. If $k \equiv 23(\bmod 25)$ then the invariants both vanish; otherwise for $k \equiv 3(\bmod 5)$ the invariants differ, and so distinguish these two spaces. Most interesting is the fact that for all values of $k \leqq 200$ the norm of the invariant for $L(25,4)$ is the same as the norm of the invariant for $L(25,9)$. This is an

19 We thank Kevin Walker for pointing out this example 
Table 1. Witten invariants for $L(5, q)$

\begin{tabular}{rrrrr}
\hline$k$ & $L(5,1)$ & $L(5,2)$ & $L(5.3)$ & $L(5,4)$ \\
\hline 1 & 0.707107 & -0.707107 & -0.707107 & 0.707107 \\
2 & $0.500000 i$ & 0.500000 & 0.500000 & $-0.500000 i$ \\
3 & $-0.415627+0.572061 i$ & 0 & 0 & $-0.415627-0.572061 i$ \\
4 & -0.288675 & -0.288675 & -0.288675 & -0.288675 \\
5 & $0.115960-0.508055 i$ & 0.521121 & 0.521121 & $0.115960+0.508055 i$ \\
6 & $0.326641-0.326641 i$ & -0.461940 & -0.461940 & $0.326641+0.326641 i$ \\
7 & $0.151506+0.262417 i$ & +0.300013 & 0.303113 & $0.151506-0.262417 i$ \\
8 & $-0.154508+0.475528 i$ & 0 & 0 & $-0.154508-0.475528 i$ \\
9 & $-0.221192+0.064948 i$ & -0.230530 & -0.230530 & $-0.221192-0.064948 i$ \\
10 & $-0.394338 i$ & 0.394338 & 0.394338 & $0.394338 i$ \\
11 & $0.208334-0.301824 i$ & -0.366744 & -0.366744 & $0.208334+0.301824 i$ \\
12 & $0.146930+0.184244 i$ & 0.235657 & 0.235657 & $0.146930-0.184244 i$ \\
13 & $-0.084880+0.399327 i$ & 0 & 0 & $-0.084880-0.399327 i$ \\
14 & $-0.181472+0.075168 i$ & -0.196424 & -0.196424 & $-0.181472-0.075168 i$ \\
15 & $-0.030440-0.328496 i$ & 0.329903 & 0.329903 & $-0.030440+0.328496 i$ \\
16 & $0.156615-0.271266 i$ & -0.313231 & -0.313231 & $0.156615+0.271266 i$ \\
17 & $0.134967+0.146613 i$ & 0.199277 & 0.199277 & $0.134967-0.146613 i$ \\
18 & $-0.055308+0.349201 i$ & 0 & 0 & $-0.055308-0.349201 i$ \\
19 & $-0.156628+0.075428 i$ & -0.173844 & -0.173844 & $-0.156628-0.075428 i$ \\
20 & $-0.041171-0.286353 i$ & 0.289298 & 0.289298 & $-0.041171+0.286353 i$ \\
291 & $0.025223-0.074359 i$ & -0.078520 & -0.078520 & $0.025223+0.074359 i$ \\
292 & $0.038966+0.029081 i$ & 0.048622 & 0.048622 & $0.038966-0.029081 i$ \\
293 & $-0.000980+0.092052 i$ & 0 & 0 & $-0.000980-0.092052 i$ \\
294 & $-0.039331+0.027817 i$ & -0.048174 & -0.048174 & $-0.039331-0.027817 i$ \\
295 & $-0.023188-0.074576 i$ & 0.078098 & 0.078098 & $-0.023188+0.074576 i$ \\
296 & $0.024994-0.073739 i$ & -0.077860 & -0.077860 & $0.024994+0.073739 i$ \\
297 & $0.038643+0.028827 i$ & 0.048211 & 0.048211 & $0.038643-0.028827 i$ \\
298 & $-0.000955+0.091282 i$ & 0 & 0 & $-0.000955-0.091282 i$ \\
299 & $-0.038999+0.027595 i$ & -0.047774 & -0.047774 & $-0.038999-0.027595 i$ \\
300 & $-0.023011-0.073950 i$ & 0.077448 & 0.077448 & $-0.023011+0.073950 i$ \\
\hline & & & &
\end{tabular}

example of two distinct spaces which are not distinguished by the norm of the Witten invariant. We studied several other pairs of homotopy equivalent lens spaces and observed in these examples that if the norm of the invariant distinguishes the spaces, then one of the invariants is zero.

Table 4 shows some values of the invariant for $L(30, q)$. This illustrates a larger fundamental group which is not of prime order. The invariant vanishes for $k$ odd. Notice the diffeomorphism $L(30,7) \approx L(30,13)$. Also, we have not printed the values of the invariant for $L(30,17), L(30,19), L(30,23)$, and $L(30,29)$, since these four spaces are diffeomorphic to the four displayed, but with the opposite orientation. As expected, the invariants are the conjugates of the ones displayed in the table.

For the lens spaces $L(p, \pm 1)$ we have an explicit formula for the Witten invariant at level $k$ :

$$
Z_{L(p, \pm 1)}(k)=\left(\frac{2}{k+2}\right) e^{2 \pi i\left( \pm \frac{3 k}{8(k+2)}\right)} \sum_{\alpha=1}^{k+1} \sin ^{2}\left(\frac{\alpha \pi}{k+2}\right) e^{2 \pi i\left(\frac{\alpha^{2}-1}{4(k+2)}\right)(\mp p)}
$$

[This is the $(1,1)$ entry of the matrix $\widetilde{S} \widetilde{T}^{\mp p} \widetilde{S}$ multiplied by a correction factor for the framing.] We use (4.1) in the large $k$ computations below. 
Table 2. Witten invariants for $L(7, q)$

\begin{tabular}{|c|c|c|c|}
\hline$k$ & $L(7,1)$ & $L(7,2)$ & $\begin{array}{l}\text { Distinguishes } \\
\text { without framing }\end{array}$ \\
\hline 1 & $0.707107 i$ & $0.707107 i$ & \\
\hline 2 & $0.353553-0.353553 i$ & $-0.353553+0.353553 i$ & \\
\hline 3 & $0.601501 i$ & $-0.353553-0.486624 i$ & \\
\hline 4 & 0.288675 & 0.288675 & \\
\hline 5 & $-0.552838+0.440874 i$ & 0 & \\
\hline 6 & $0.073223-0.176777 i$ & $-0.176777-0.073223 i$ & \\
\hline 7 & $-0.402046-0.232121 i$ & $0.402046-0.232121 i$ & \\
\hline 8 & 0.361803 & $0.111803+0.344095 i$ & \\
\hline 9 & $-0.090789-0.309199 i$ & $-0.174223+0.271096 i$ & \\
\hline 10 & $0.278839+0.278839 i$ & $-0.278839-0.278839 i$ & \\
\hline 11 & $0.150013-0.103547 i$ & $0.180950-0.021971 i$ & \\
\hline 12 & $-0.216942+0.450484 i$ & 0 & \\
\hline 13 & $-0.148519 i$ & $-0.141250-0.045895 i$ & \\
\hline 14 & $-0.340097-0.067650 i$ & $0.288320-0.192650 i$ & \\
\hline 15 & $0.263269-0.074906 i$ & $0.098878+0.255234 i$ & \\
\hline 16 & $-0.127674-0.221138 i$ & $-0.127674+0.221138 i$ & \\
\hline 17 & $0.263301+0.172023 i$ & $-0.231396-0.213015 i$ & \\
\hline 18 & $0.101515-0.101515 i$ & $0.141797-0.022458 i$ & \\
\hline 19 & $-0.120333+0.390111 i$ & 0 & \\
\hline 20 & $-0.017825-0.123977 i$ & $-0.120179-0.035288 i$ & \\
\hline
\end{tabular}

Table 3. Witten invariants for $L(25,4)$ and $L(25,9)$

\begin{tabular}{rcl}
\hline$k$ & $L(25,4)$ & \multicolumn{1}{c}{$L(25,9)$} \\
\hline 1 & 0.707107 & 0.707107 \\
2 & 0.500000 & 0.500000 \\
3 & $-0.415627-0.572061 i$ & $0.672499+0.218508 i$ \\
4 & 0.288675 & 0.288675 \\
5 & $0.376521-0.181323 i$ & $0.376521-0.181323 i$ \\
6 & 0.191342 & 0.191342 \\
7 & $0.232121-0.402046 i$ & $0.232121-0.402046 i$ \\
8 & $-0.154508-0.475528 i$ & $0.404508-0.293893 i$ \\
9 & $0.254000-0.293131 i$ & $0.254000-0.293131 i$ \\
10 & 0.105662 & 0.105662 \\
11 & $-0.083115-0.043622 i$ & $-0.083115-0.043622 i$ \\
12 & $-0.075776-0.331996 i$ & $-0.075776-0.331996 i$ \\
13 & $0.303388-0.273171 i$ & $-0.166050-0.372953 i$ \\
14 & $-0.346760 i$ & $-0.346760 i$ \\
15 & $-0.105346-0.065228 i$ & $-0.105346-0.065228 i$ \\
16 & $-0.127674-0.221138 i$ & $-0.127674-0.221138 i$ \\
17 & $-0.121857-0.094845 i$ & $-0.121857-0.094845 i$ \\
18 & $0.349201-0.055308 i$ & $-0.315018-0.160510 i$ \\
19 & $-0.130874-0.164111 i$ & $-0.130874-0.164111 i$ \\
20 & $-0.105369-0.230726 i$ & $-0.105369-0.230726 i$ \\
\hline
\end{tabular}

Seifert Spaces. The invariant for Seifert spaces is computed by combining (1.17) with (2.2). The canonical framing is determined using (2.4) and (2.7).

Table 5 displays the invariant for the homology spheres $\Sigma(2,3,6 n+1)$. Notice that $\Sigma(2,3,1) \approx S^{3}$. We checked that the values computed using the Seifert formula agree with those calculated directly from (1.13). The new phenomenon here is a 
Table 4. Witten invariants for $L(30, q)$

\begin{tabular}{rrcrc}
\hline$k$ & $L(30,1)$ & $L(30,7)$ & \multicolumn{1}{c}{$L(30,11)$} & \multicolumn{1}{c}{$L(30,13)$} \\
\hline 2 & $-0.270+0.270 i$ & $-0.270-0.270 i$ & $-0.270-0.270 i$ & $-0.270-0.270 i$ \\
4 & $-0.500+0.500 i$ & $-0.500+0.500 i$ & $-0.500-0.500 i$ & $-0.500+0.500 i$ \\
6 & $-0.127-0.052 i$ & $-0.127+0.052 i$ & $0.127-0.052 i$ & $-0.127+0.052 i$ \\
8 & $0.321+0.630 i$ & 0 & $-0.500+0.500 i$ & 0 \\
10 & $-0.191+0.461 i$ & $0.461+0.191 i$ & $-0.191-0.461 i$ & $0.461+0.191 i$ \\
92 & $0.002-0.083 i$ & $-0.072-0.042 i$ & $-0.082-0.011 i$ & $-0.072-0.042 i$ \\
94 & $-0.075+0.159 i$ & $-0.105+0.141 i$ & $-0.166-0.059 i$ & $-0.105+0.141 i$ \\
96 & $0.009-0.040 i$ & $-0.036+0.020 i$ & $0.031-0.027 i$ & $-0.036+0.020 i$ \\
98 & $-0.203-0.092 i$ & 0 & $-0.116+0.190 i$ & 0 \\
100 & $0.069-0.156 i$ & $0.162+0.054 i$ & $-0.096-0.141 i$ & $0.162+0.054 i$ \\
\hline
\end{tabular}

Tab. 5. Witten invariants for $\Sigma(2,3,6 n+1)$

\begin{tabular}{clccr}
\hline$k$ & $\Sigma(2,3,1)$ & $\Sigma(2,3,7)$ & $\Sigma(2,3,13)$ & \multicolumn{1}{c}{$\Sigma(2,3,19)$} \\
& $\Sigma(2,3,25)$ & $\Sigma(2,3,31)$ & $\Sigma(2,3,37)$ & $\Sigma(2,3,43)$ \\
\hline 1 & 0.707 & 0.707 & 0.707 & 0.707 \\
& 0.707 & 0.707 & 0.707 & 0.707 \\
2 & 0.500 & -0.500 & 0.500 & -0.500 \\
& 0.500 & -0.500 & 0.500 & -0.500 \\
3 & 0.372 & $-0.716-0.354 i$ & $0.372-0.707 i$ & $-0.301+0.219 i$ \\
& $-0.301-0.926 i$ & 0.372 & $-0.716-0.354 i$ & $0.372-0.707 i$ \\
4 & 0.289 & 0.289 & 0.289 & 0.289 \\
& 0.289 & 0.289 & 0.289 & 0.289 \\
5 & 0.232 & $0.585+0.734 i$ & $-0.052+0.226 i$ & $0.145-0.835 i$ \\
& $0.428-0.407 i$ & $-0.492+0.327 i$ & $-0.846-0.045 i$ & 0.232 \\
6 & 0.191 & $0.191 i$ & -0.733 & $-0.733 i$ \\
& 0.191 & $0.191 i$ & -0.733 & $-0.733 i$ \\
7 & 0.161 & $0.099-0.878 i$ & $-0.176+0.402 i$ & 0.161 \\
& $0.099-0.878 i$ & $-0.176+0.402 i$ & 0.161 & $0.099-0.878 i$ \\
8 & 0.138 & $0.388-0.507 i$ & $-0.362+0.526 i$ & $0.043+0.131 i$ \\
& $-0.766-0.557 i$ & 0.138 & $0.388-0.507 i$ & $-0.362+0.526 i$ \\
9 & 0.120 & $-0.365+0.407 i$ & $-0.758+0.512 i$ & $-0.700+0.035 i$ \\
& $-0.258-0.651 i$ & $0.151-0.902 i$ & $0.218-0.501 i$ & $0.050+0.109 i$ \\
10 & 0.106 & $-0.789+0.183 i$ & 0.106 & $-0.789+0.183 i$ \\
& 0.106 & $-0.789+0.183 i$ & 0.106 & $-0.789+0.183 i$ \\
\hline
\end{tabular}

certain periodicity (2.28), which occurs since $\Sigma(2,3,6 n+1)$ is $-1 / n$ surgery on the right-handed trefoil knot. The periodicity allows us to determine from the table some Witten invariants of $\Sigma(2,3,6 n \pm 1)$ which are not explicitly given. In particular, we obtain $\Sigma(2,3,6 n-1)$ for $n>0$ by noting that this is the same as $\Sigma(2,3,-6 n+1)$ with reversed orientation, or $-1 / n$ surgery on the left trefoil. [Recall our orientation convention preceding (2.10).] Thus, the Poincaré homology sphere $\Sigma(2,3,5)$ would appear (with reversed orientation) just to the left of the $n=0$ column. From the table we may now immediately verify (for most values of $n)$ the known fact that $\Sigma(2,3,6 n \pm 1)$ cannot admit an orientation reversing selfdiffeomorphism unless $n=0$. For example, $k=3$ proves this for $n \neq 0(\bmod 5)$ and $k=5$ works for $n \neq 0(\bmod 7)$. It seems likely that all values of $k$ together should prove the fact in general, and furthermore should distinguish all $\Sigma(2,3,6 n \pm 1)$. 
Surgery on the Trefoil. Recall from (2.11) that $p$-surgery on the left trefoil is

$$
X_{\text {tref }}(p)=X(-2 / 1,3 / 1,(p+6) / 1) .
$$

We have already listed some Witten invariants of $X_{\text {tref }}(1)=\Sigma(2,3,7)$ (with reversed orientation) and $X_{\text {tref }}(-1)=\Sigma(2,3,5)$. Table 6 displays the Witten invariants of $X_{\text {tref }}(p)$ for $p=-2,0,2$ at low values of $k$. The computations are analogous to those for $\Sigma(2,3,6 n+1)$. Note that the invariant distinguishes the handedness of the trefoil: If we reverse the orientation of $X_{\text {tref }}(p)$ we obtain $-p$-surgery on the right trefoil, but the invariants for $X_{\text {tref }}(p)$ are usually not conjugate to those for $X_{\text {tref }}(-p)$. In particular, the invariants for $X_{\text {tref }}(0)$ are usually not real.

Since $X_{\text {tref }}(0)$ is a torus bundle over a circle, we tested this data against the predicted formula (1.24) of Quantum Field Theory. The monodromy matrix is (2.12). The 2-framing inherited by $X_{\text {tref }}(0)$ as a torus bundle with this monodromy can be converted to the canonical 2 -framing by adding -4 twists [cf. the discussion following (2.12)], so we corrected (1.24) by a factor of $e^{2 \pi i c / 6}$, in accordance with (1.4). The resulting numbers (for $1 \leqq k \leqq 20$ ) checked against the values for $X_{\text {tref }}(0)$ computed as above.

We tested Witten's assertion (1.23) that the Chern-Simons theory reproduces the Jones polynomial on the left-handed trefoil in $S^{3}$. The value of the Jones polynomial was already given in (1.21). To compute the Witten invariant we carry out the surgery procedure described after (2.13). Then we apply the gluing law as in (1.17), but now just carry along the extra circle, which after surgery becomes the left trefoil with the -6 framing. Thus the formula for its Witten invariant (without a correction for the framing) is

$$
Z_{\left(S^{3} ; \text { tref }\right)}=\sum_{\alpha_{1}, \alpha_{2}, \beta}\left(\tilde{M}_{1}\right)_{1}^{\alpha_{1}}\left(\tilde{M}_{2}\right)_{1}^{\alpha_{2}} \frac{\tilde{S}_{\alpha_{1} \beta} \tilde{S}_{\alpha_{2} \beta} \tilde{S}_{2 \beta}}{\left(\tilde{S}_{1 \beta}\right)},
$$

\begin{tabular}{|c|c|c|c|}
\hline$k$ & $p=-2$ & $p=0$ & $p=2$ \\
\hline 1 & 0 & 1.000000 & 0 \\
\hline 2 & $0.923880 \mathrm{i}$ & 0 & $-0.923880 i$ \\
\hline 3 & 0 & $-0.309017+0.951057 i$ & 0 \\
\hline 4 & $-0.288675+0.866025 i$ & $0.500000+0.866025 i$ & $-0.288675+0.866025 i$ \\
\hline 5 & 0 & 0 & 0 \\
\hline 6 & $-0.310153+0.865723 i$ & $-0.707107+0.707107 i$ & $0.670633-0.670633 i$ \\
\hline 7 & 0 & $0.173648+0.984808 i$ & 0 \\
\hline 8 & $-0.232292+0.903112 i$ & 0 & $-0.720289+0.535941 i$ \\
\hline 9 & 0 & $-0.841254+0.540641 i$ & 0 \\
\hline 10 & $-0.099046+0.942055 i$ & $1.000000 i$ & $0.892399-0.333293 i$ \\
\hline 11 & 0 & 0 & 0 \\
\hline 12 & $0.069468+0.959316 i$ & $-0.900969+0.433884 i$ & $-0.873783+0.198082 i$ \\
\hline 13 & 0 & $-0.104528+0.994522 i$ & 0 \\
\hline 14 & $0.257802+0.940545 i$ & 0 & $0.950894-0.014598 i$ \\
\hline 15 & 0 & $-0.932472+0.361242 i$ & 0 \\
\hline 16 & $0.451105+0.877817 i$ & $-0.173648+0.984808 i$ & $-0.891344-0.113566 i$ \\
\hline 17 & 0 & 0 & 0 \\
\hline 18 & $0.634633+0.768530 i$ & $-0.951057+0.309017 i$ & $0.906578+0.274378 i$ \\
\hline 19 & 0 & $-0.222521+0.974928 i$ & 0 \\
\hline 20 & $0.794263+0.614787 i$ & 0 & $-0.815103-0.388241 i$ \\
\hline
\end{tabular}

Table 6. Witten invariants for $p$-surgery on the left trefoil 
where $\tilde{M}_{1}$ and $\tilde{M}_{2}$ are representatives of the matrices (2.1) for $p_{i}=-2,3$, respectively. Finally, we correct for the canonical 2-framing of $S^{3}$ using (2.4), (2.7) and for the normal framing of the knot using (1.22). We verified (1.23) for $1 \leqq k \leqq 50$.

Small $k$. Reshetikhin and Turaev [RT] construct an invariant $\varrho_{X}$ of an oriented 3-manifold $X$ which they suggest is equal to Witten's invariant, ${ }^{20}$ but they provide no proof. ${ }^{21}$ The formula for the invariant is quite complicated. On the other hand, after much work Kirby and Melvin [KM1] were able to simplify this expression for low values of $k$. Their formulas, which we recount below, are in terms of elementary topological invariants and the Jones polynomial. We checked our numbers against the $\mathrm{KM}$ formulas for $k=1,2,3,4$, and we summarize our results in this section. Our experiments not only support the hypothesis that the RT invariant (as adjusted by KM) is equal to Witten's invariant, ${ }^{22}$ but also suggest that the RT invariant uses the canonical framing. (That the canonical framing enters into the work of Reshetikhin and Turaev was anticipated by Atiyah [A1].)

The KM formulas apply to an oriented 3-manifold $X=X_{L}$ obtained by integer surgery on a link $L$. As in Sect. 2 we denote the corresponding 4-manifold by $W=W_{L}$. Then for $k=1$ Kirby and Melvin compute

$$
\varrho_{X}(1)=2^{-(n+1) / 2} e^{-2 \pi i(\sigma / 8)} \sum_{S<L} i^{s \cdot s},
$$

where $n$ is the number of components of the link, $\sigma$ is the signature of $W$, the summation is over all sublinks of the given link, and $S \cdot S$ is the sum of the entries in the linking matrix of $S$. If $X$ has a unique spin structure (i.e., if $X$ is a $\mathbb{Z} / 2$-homology sphere), then this reduces to

$$
\varrho_{X}(1)= \pm e^{-2 \pi i(\mu(X) / 8)} / \sqrt{2}
$$

where $\mu(X)$ is the (unique) mu invariant of $X$, and the sign is positive if $\left|H_{1}(X)\right| \equiv \pm 1(\bmod 8)$ and negative otherwise. For $k=2$ the $\mathrm{KM}$ formula is

$$
\varrho_{X}(2)=\frac{1}{2} \sum_{\Theta} e^{-2 \pi i(3 \mu(X, \Theta) / 16)},
$$

where the summation runs over the spin structures $\Theta$ on $X$.

We verified $Z_{X}(k)=\varrho_{X}(k), k=1,2$ for a variety of spaces. First, we checked the lens spaces $L(p, q)$ for $2 \leqq p \leqq 20$. The mu invariants for lens spaces are computed in (2.14) and (2.15). We can easily check the homology spheres $\Sigma(2,3,6 n \pm 1)$ for all $n$, using Eq. (2.16) for the unique mu invariant $\mu(\Sigma(2,3,6 n \pm 1)) \equiv 8 n(\bmod 16)$ and the periodicity described above. The verification for small values of $n$ can be read off from Table 5. We also checked the manifolds $X_{\text {tref }}(p)$ for $-10 \leqq p \leqq 10$. Here we need the mu invariant formula (2.17).

The KM formulas for $k=3,4$ only apply to knots (i.e., links with one component). Let $p$ denote the surgery coefficient. The formula for $k=3$ only applies

\footnotetext{
${ }^{20}$ However, the RT invariant (after KM) is normalized to be 1 on the 3 -sphere. In the formulaes below we correct by the factor (1.13) to normalize according to quantum field theory. Also, KM adjusted RT's normalization so that the invariant conjugates under orientation reversal

${ }^{21}$ Witten's invariant depends on a positive integer $k$. The RT invariant (in the notation of [KM1]) depends on a root of unity $e^{2 \pi i / r}$. The correspondence is $r=k+2$. For simplicity we will use the notation $\varrho_{X}(k)$ for the (renormalized) RT invariant

${ }_{22}$ As mentioned in the introduction, Melvin has verified this for the class of manifolds plumbings on trees - considered in this paper

Added in proof. Kevin Walker has now proved this for all manifolds.
} 
to $p>0$. After some algebraic simplifications, their formula is

$$
\varrho_{X}(3)=\frac{2}{5} \sin ^{2}\left(\frac{\pi}{5}\right) e^{-2 \pi i(9 / 40)}\left[1+(-i)^{p}+4\left(1+i^{p}\right) \cos ^{2}\left(\frac{\pi}{5}\right) e^{2 \pi i(3 p / 20)} \widetilde{V}_{L}\left(e^{2 \pi i / 5}\right)\right],
$$

where $\tilde{V}_{L}$ is the Jones polynomial (1.20) of the knot $L$. The formula for $k=4$ applies to any integer $p$ :

$$
\varrho_{X}(4)=\frac{(-i)^{\operatorname{sign}(p)}}{6}\left[1+2 e^{2 \pi i(p / 3)}+\frac{3}{2}\left(1+(-1)^{p}\right) e^{2 \pi i(p / 8)} \tilde{V}_{L}\left(e^{2 \pi i / 6}\right)\right]
$$

We verified $Z_{X}(k)=\varrho_{X}(k), k=3$ for $L(p,-1)$ and for $k=4$ we checked $L(p, \pm 1)$. In this case $\widetilde{V}_{L}=1$. We also carried out this verification for the surgeries in the trefoil $X_{\text {tref }}(p)$. For $k=3$ we checked $1 \leqq p \leqq 10$ and for $k=4$ we checked $-10 \leqq p \leqq 10$. Here we used Eq. (1.21) for the Jones polynomial $\widetilde{V}_{L}$.

Large $k$. We compare our exact calculations for $L(p, q)$ and some homology spheres $\Sigma\left(p_{1}, p_{2}, p_{3}\right)$ with the asymptotic behavior $(1.36)$ predicted by the path integral. We begin with the lens spaces $L(p, \pm 1), p \geqq 3$, for which we have the explicit formula (4.1) for all $k$. In Appendix A, we give a (partial) proof of the asymptotic formula

$$
Z_{L(p, \pm 1)}(k) \sim \pm \frac{1}{2} \sum_{n=1}^{\left[\frac{p-1}{2}\right]} \sqrt{2}\left(\frac{4}{\sqrt{p}} \sin ^{2}\left(\frac{2 n \pi}{p}\right)\right) i e^{ \pm 2 \pi i\left(\frac{n^{2}}{p}\right)(k+2)}(k+2)^{-1 / 2}
$$

as $k \rightarrow \infty$. Except for the factor of $\sqrt{2}$, this agrees with the predicted result. The torsion is (2.25) with $q^{*}= \pm 1$. The Chern-Simons invariant is computed from (2.20). Finally, we claim that for $L(p, \pm 1)$ we have the spectral flow

$$
I_{A_{n}} \equiv \pm 1 \quad(\bmod 4)
$$

for each of the flat connections. We do not provide a proof, but assert this on the basis of numerical calculations from (2.21). The fact that $\operatorname{dim} H^{0}\left(A_{n}\right)=1$ for each flat connection accounts for the factor $(k+2)^{-1 / 2}$. Recall that the torsion $\tau_{L(p, q)}\left(A_{n}\right)$ depends on a basis choice, since the cohomology is nonzero, so the unexplained factor of $\sqrt{2}$ does not contradict the path integral. This factor appears in all of our lens space results.

Lisa Jeffrey $[\mathrm{J}]$ uses a reciprocity law to rewrite (4.1):

$$
\begin{aligned}
Z_{L(p, \pm 1)}(k)= & \pm \frac{1}{4} e^{ \pm 2 \pi i\left(\frac{p-3}{4(k+2)}\right)} \sum_{n=1}^{p} \sqrt{2}\left(\frac{2}{\sqrt{p}}\left[1-e^{ \pm \frac{2 \pi i}{p(k+2)}} \cos \left(\frac{4 n \pi}{p}\right)\right]\right) \\
& \times i e^{ \pm 2 \pi i\left(\frac{n^{2}}{p}\right)(k+2)}(k+2)^{-1 / 2}
\end{aligned}
$$

The leading order asymptotics (4.2) follow easily from (4.4). One could also obtain a full asymptotic expansion from (4.4). Jeffrey's results cover other $L(p, q)$ as well.

For other lens spaces we verified the asymptotic expansion numerically. We carried this out for $L(5,2), L(7,2), L(7,3), L(8,3)$, and $L(12,5)$. In all cases, up to the 
Table 7. Asymptotic values of the Witten invariant for $L(12,5)$

\begin{tabular}{cccl}
\hline$k$ & Exact value & Asymptotic value & \multicolumn{1}{c}{ Ratio } \\
\hline 191 & $-0.032268+0.018630 i$ & $-0.032080+0.018579 i$ & $0.994936-0.001350 i$ \\
192 & 0.101535 & 0.101535 & 1.000000 \\
193 & $-0.087706-0.087706 i$ & $-0.087706-0.087706 i$ & 1.000000 \\
194 & $-0.050508+0.087482 i$ & $-0.050274+0.087616 i$ & $0.999996-0.002671 i$ \\
195 & $0.119199+0.068819 i$ & $0.119247+0.068636 i$ & $0.999642-0.001328 i$ \\
196 & $-0.174078 i$ & $-0.174078 i$ & 1.000000 \\
197 & $-0.118598+0.068473 i$ & $-0.118550+0.068653 i$ & $1.000351-0.001316 i$ \\
198 & $0.050000+0.086603 i$ & $0.050227+0.086471 i$ & $0.999997-0.002618 i$ \\
199 & $0.086387-0.086387 i$ & $0.086387-0.086387 i$ & 1.000000 \\
200 & -0.099503 & -0.099503 & 1.000000 \\
\hline
\end{tabular}

mysterious factor $\sqrt{2}$, the exact results are asymptotic (numerically) to the predicted value (1.36). For example, when $X=L(12,5)$ we verify

$$
\begin{aligned}
Z_{L(12,5)}(k) \sim \frac{i}{2} \frac{\sqrt{2}}{\sqrt{12 \cdot(k+2)}}\{ & 4 \sin ^{2}\left(\frac{2}{12} \pi\right) \mathrm{e}^{2 \pi i\left(\frac{5}{12}\right)(k+2)} \\
& -4 \sin ^{2}\left(\frac{4}{12} \pi\right) e^{2 \pi i\left(\frac{8}{12}\right)(k+2)} \\
& +4 \sin ^{2}\left(\frac{6}{12} \pi\right) e^{2 \pi i\left(\frac{9}{12}\right)(k+2)} \\
& -4 \sin ^{2}\left(\frac{4}{12} \pi\right) e^{2 \pi i\left(\frac{8}{12}\right)(k+2)} \\
& \left.+4 \sin ^{2}\left(\frac{2}{12} \pi\right) e^{2 \pi i\left(\frac{5}{12}\right)(k+2)}\right\} .
\end{aligned}
$$

We illustrate our numerical check in Table 7, where we list the exact value of the Witten invariant computed using (1.19), the asymptotic value predicted by (4.5), and the ratio for $191 \leqq k \leqq 200$. We also illustrate these results graphically in Figs. 8 and 9, where we plot the Witten invariant and its norm for the lens spaces $L(5,1)$ and $L(12,5)$.

We can check the asymptotic formula (1.32) more precisely for the Brieskorn homology spheres $\Sigma\left(p_{1}, p_{2}, p_{3}\right)$, since the equivalence classes of flat connections are isolated and have trivial cohomology. As we discussed in Sect. 2 such an equivalence class of flat connections corresponds to a triple of integers $\left\langle l_{1}, l_{2}, l_{3}\right\rangle$. We ran checks on several homology spheres in the family (2.10). For the Poincare homology sphere $X=\Sigma(2,3,5)$ there are two flat connections, and they correspond to the triples $A_{1}=\langle 1,1,1\rangle$ and $A_{2}=\langle 1,1,3\rangle .{ }^{23}$ From (2.22) we compute the Chern-Simons invariants

$$
S_{X}\left(A_{1}\right) \equiv \frac{-1}{120} \quad(\bmod 1), \quad S_{X}\left(A_{2}\right) \equiv \frac{-49}{120} \quad(\bmod 1) .
$$

${ }^{23}$ In this case the fundamental group is finite, the binary icosohedral group of order 120 

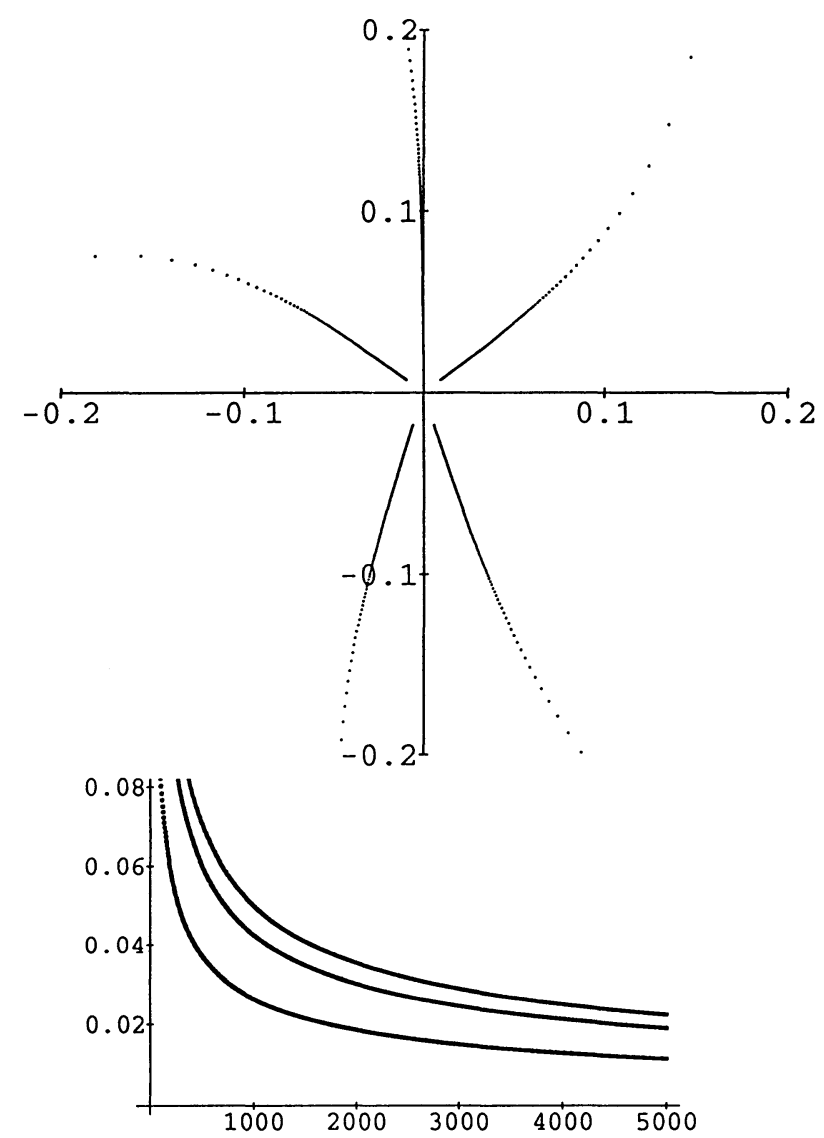

Fig. 8. Witten invariants for $L(5,1): 1 \leqq k \leqq 5000$

The spectral flows

$$
I_{A_{1}} \equiv 4 \quad(\bmod 8), \quad I_{A_{2}} \equiv 0 \quad(\bmod 8),
$$

are computed from (2.23). The torsions are given by (2.26) and are

$$
\tau_{X}\left(A_{1}\right)=\frac{8}{5} \sin ^{2}\left(\frac{\pi}{5}\right), \quad \tau_{X}\left(A_{2}\right)=\frac{8}{5} \sin ^{2}\left(\frac{2 \pi}{5}\right) .
$$

Therefore, (1.32) yields the asymptotic formula

$$
Z_{\Sigma(2,3,5)}(k) \sim \sqrt{\frac{2}{5}} e^{-3 \pi i / 4}\left\{\sin \left(\frac{\pi}{5}\right) e^{-\pi i(k+2) / 60}+\sin \left(\frac{2 \pi}{5}\right) e^{-49 \pi i(k+2) / 60}\right\} .
$$

In Table 8 we display some numerical data for $191 \leqq k \leqq 200$ comparing the exact value and the asymptotic value predicted by (4.6). The agreement between these numbers strongly supports the validity of the asymptotic expansion. In Fig. 10 we graph the exact value of $Z_{\Sigma(2,3,5)}(k)$ for $1 \leqq k \leqq 200$.

We obtained similar results for $\Sigma(2,3,7), \Sigma(2,3,11), \Sigma(2,3,17)$, and $\Sigma(2,3,19)$. The space $X=\Sigma(2,3,17)$ illustrates the importance of the spectral flow term in (1.32). Here there are 6 classes of flat connections, labelled by $A_{1}=\langle 1,1,3\rangle, A_{2}=\langle 1,1,5\rangle$, 

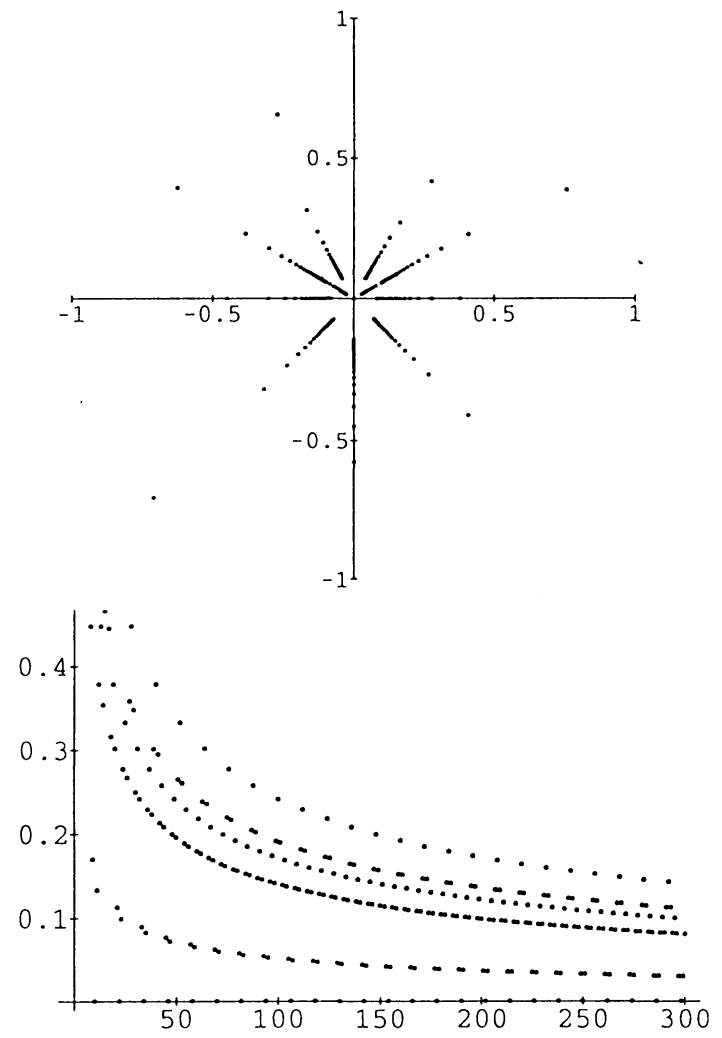

Fig. 9. Witten invariants for $L(12,5): 1 \leqq k \leqq 300$

Table 8. Asymptotic values of the Witten invariant for $\Sigma(2,3,5)$

\begin{tabular}{crrc}
\hline$k$ & Exact value & Asymptotic value & - \\
\hline 191 & $0.590150-0.540484 \mathrm{i}$ & $0.614364-0.510640 \mathrm{i}$ & $1.000570-0.048104 i$ \\
192 & $-0.114537+0.352848 i$ & $-0.133223+0.347057 i$ & $0.996531-0.052510 i$ \\
193 & $0.973668-0.047578 i$ & 0.973249 & $1.000430-0.048886 i$ \\
194 & $-0.148285-0.340505 i$ & $-0.133223-0.347057 i$ & $0.998066-0.044143 i$ \\
195 & $0.639755+0.480216 i$ & $0.614364+0.510640 i$ & $1.000100-0.049604 i$ \\
196 & $0.431065-0.673858 i$ & $0.461267-0.652250 i$ & $1.000256-0.046485 i$ \\
197 & $-0.019691+0.370860 i$ & $-0.038858+0.369712 i$ & $0.997683-0.051601 i$ \\
198 & $0.928612-0.296421 i$ & $0.940086-0.251895 i$ & $1.000453-0.047243 i$ \\
199 & $-0.230939-0.290393 i$ & $-0.218508-0.300750 i$ & $0.997114-0.043429 i$ \\
200 & $0.741961+0.299562 i$ & $0.725594+0.334231 i$ & $1.000453-0.047990 i$ \\
\hline
\end{tabular}

$A_{3}=\langle 1,1,7\rangle, A_{4}=\langle 1,1,9\rangle, A_{5}=\langle 1,1,11\rangle$, and $A_{6}=\langle 1,2,4\rangle$. The ordered list of Chern-Simons invariants is

$$
\left\{S_{X}\left(A_{i}\right)\right\} \equiv\left\{\frac{-1}{408}, \frac{-169}{408}, \frac{-217}{408}, \frac{-145}{408}, \frac{-361}{408}, \frac{-49}{408}\right\} \quad(\bmod 1) ;
$$

the ordered list of spectral flows is

$$
\left\{I_{A_{i}}\right\} \equiv\{4,0,6,0,4,2\} \quad(\bmod 8) ;
$$




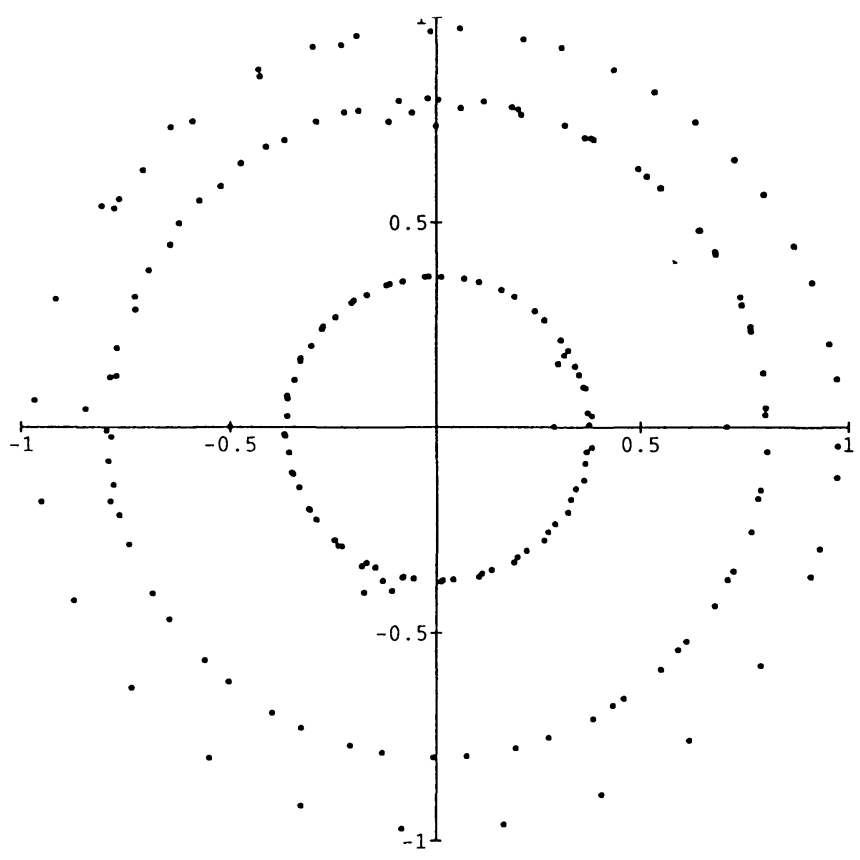

Fig. 10. Witten invariants for $\Sigma(2,3,5): 1 \leqq k \leqq 200$

and the ordered list of torsions is

$$
\begin{aligned}
\left\{\tau_{X}\left(A_{i}\right)\right\}= & \left\{\frac{8}{17} \sin ^{2}\left(\frac{\pi}{17}\right), \frac{8}{17} \sin ^{2}\left(\frac{4 \pi}{17}\right), \frac{8}{17} \sin ^{2}\left(\frac{8 \pi}{17}\right),\right. \\
& \left.\frac{8}{17} \sin ^{2}\left(\frac{3 \pi}{17}\right), \frac{8}{17} \sin ^{2}\left(\frac{2 \pi}{17}\right), \frac{8}{17} \sin ^{2}\left(\frac{7 \pi}{17}\right)\right\} .
\end{aligned}
$$

The asymptotic formula is then

$$
\begin{aligned}
Z_{\Sigma(2,3,17)}(k) \sim \sqrt{\frac{2}{17}} e^{-3 \pi i / 4}\{ & \sin \left(\frac{\pi}{17}\right) e^{-\pi i(k+2) / 204} \\
& +\sin \left(\frac{4 \pi}{17}\right) e^{-169 \pi i(k+2) / 204} \\
& -\sin \left(\frac{8 \pi}{17}\right) e^{-217 \pi i(k+2) / 204} \\
& +\sin \left(\frac{3 \pi}{17}\right) e^{-145 \pi i(k+2) / 204} \\
& +\sin \left(\frac{2 \pi}{17}\right) e^{-361 \pi i(k+2) / 204} \\
& \left.-\sin \left(\frac{7 \pi}{17}\right) e^{-49 \pi i(k+2) / 204}\right\} .
\end{aligned}
$$

We illustrate the numerical data in Table 9 for $141 \leqq k \leqq 150$ and graph the exact values in Fig. 11 for $1 \leqq k \leqq 150$. 
Table 9. Asymptotic values of the Witten invariant for $\Sigma(2,3,17)$

\begin{tabular}{crrc}
\hline$k$ & \multicolumn{1}{c}{ Exact value } & \multicolumn{1}{c}{ Asymptotic value } & Ratio \\
\hline 141 & $0.607899+0.102594 i$ & $0.596099+0.151172 i$ & $0.999182-0.081285 i$ \\
142 & $-0.104966-0.151106 i$ & $-0.094614-0.157913 i$ & $0.997181-0.067244 i$ \\
143 & $0.123614-0.139016 i$ & $0.132261-0.128045 i$ & $1.007707-0.075491 i$ \\
144 & $-0.612014+0.038199 i$ & $-0.614913-0.008261 i$ & $0.994271-0.075479 i$ \\
145 & $-0.291162-0.132171 i$ & $-0.281928-0.153204 i$ & $0.993986-0.071336 i$ \\
146 & $-0.413944+0.674785 i$ & $-0.465909+0.642185 i$ & $0.994797-0.077144 i$ \\
147 & $0.400490-0.286350 i$ & $0.419276-0.254325 i$ & $1.001116-0.075706 i$ \\
148 & $-0.091879+0.669230 i$ & $-0.143660+0.661309 i$ & $0.995194-0.077257 i$ \\
149 & $0.946786-0.263649 i$ & $0.962119-0.191329 i$ & $0.999048-0.075356 i$ \\
150 & $-0.024553-0.058313 i$ & $-0.021860-0.059113 i$ & $1.002906-0.044484 i$ \\
\hline
\end{tabular}

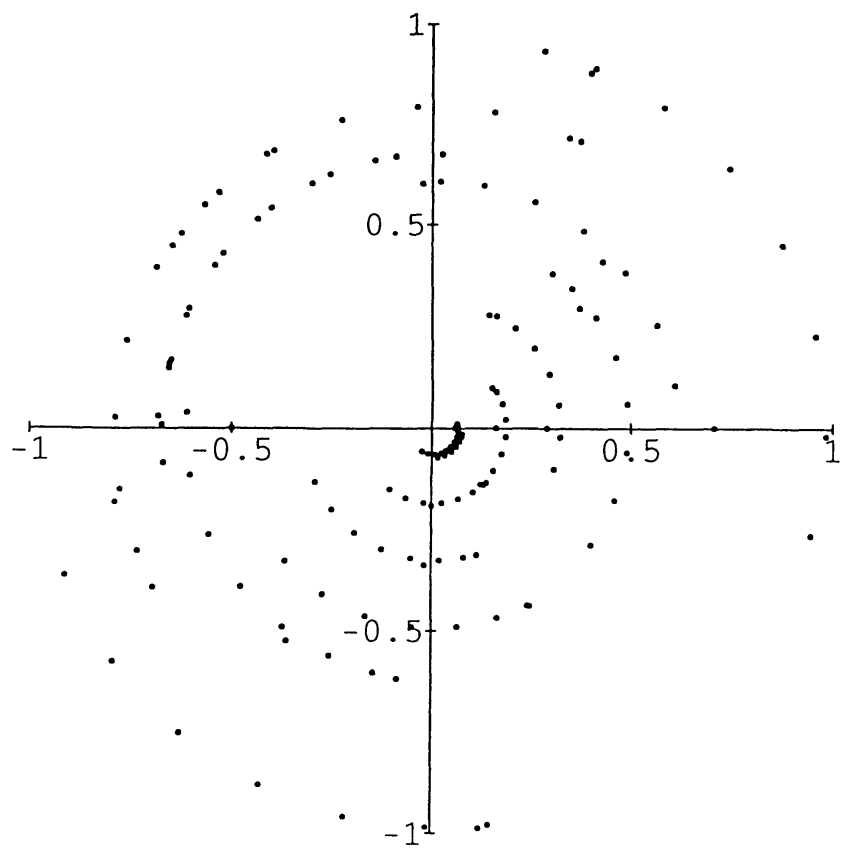

Fig. 11. Witten invariants for $\Sigma(2,3,17): 1 \leqq k \leqq 150$

We conclude this section with some qualitative data to illustrate (1.37). Our first example is the connected sum $X=\Sigma(2,3,7) \# \Sigma(2,3,7)$. We graph the Witten invariant and its norm for $1 \leqq k \leqq 175$ in Fig. 12. It is computed from the Witten invariant for $\Sigma(2,3,7)$ using (1.11). The norm exhibits the growth $\left|Z_{X}(k)\right|=O\left(k^{3 / 2}\right)$, which we checked numerically by fitting $\log \left|Z_{X}(k)\right|$ to linear functions. This behavior also follows from (1.11) using $\left|Z_{S^{3}}(k)\right|=O\left(k^{-3 / 2}\right)$ [which is (1.34)] and the fact that $\left|Z_{\Sigma(2,3,7)}\right|=O(1)$ [an experimental fact which is evident from Fig. 11 and from (1.32)]. On the other hand, it is easy to see that the equivalence classes of flat connections on $X$ come in 3 dimensional families. For a flat connection is obtained by choosing two representations of the fundamental group of $\Sigma(2,3,7)$ independently. These representations come in 3 dimensional families, so the pair of 

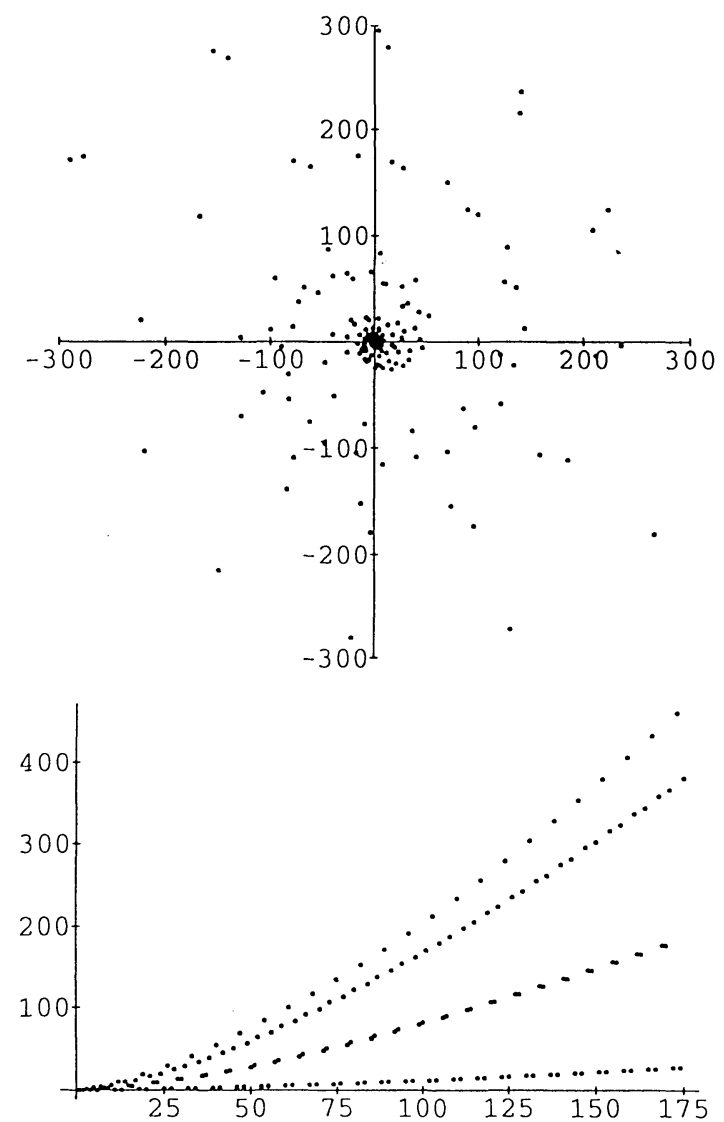

Fig. 12. Witten invariants for $\Sigma(2,3,7) \# \Sigma(2,3,7): 1 \leqq k \leqq 175$

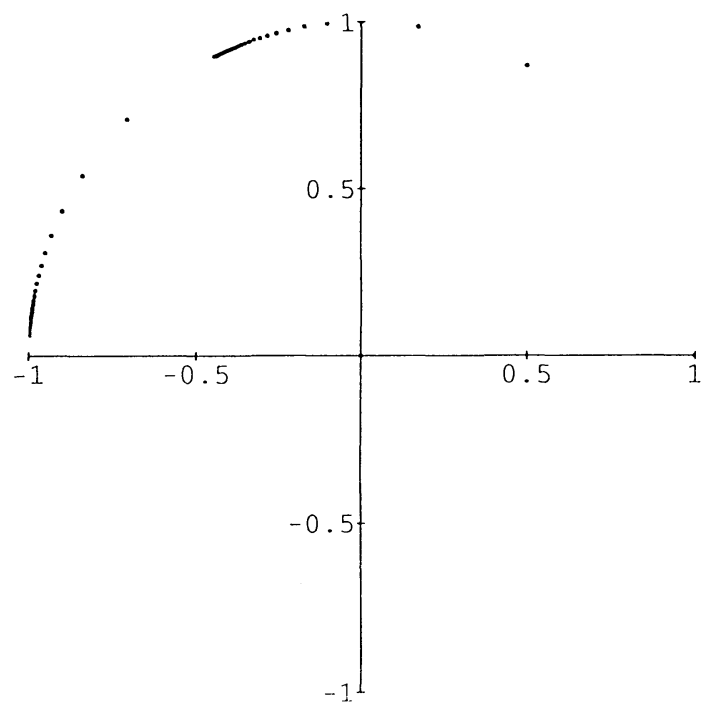

Fig. 13. Witten invariants for $X_{\text {tref }}(0): 1 \leqq k \leqq 100$ 
representations lives in a 6 dimensional family. Now the action of simultaneous conjugation reduces us back to a 3 dimensional family.

Our final example is $X=X_{\text {tref }}(0)$, which has the homology of $S^{2} \times S^{1}$. The reducible connections have $\operatorname{dim} H^{0}=\operatorname{dim} H^{1}=1$, so contribute $O(1)$ to the large $k$ behavior. In fact, it seems that $\left|Z_{X}(k)\right|=1$ for all $k \neq 2(\bmod 3)$, a fact we checked numerically for $1 \leqq k \leqq 100$. We graph the exact value of $Z_{X}(k), 1 \leqq k \leqq 100$ in Fig. 13. Notice for comparison that $Z_{S^{2} \times S^{1}}(k)=1$ for all $k$.

\section{Questions and Conclusions}

There are a few proofs in this paper which we have not been able to give completely; instead we relied on computer verifications. These proofs should be routine for those readers with appropriate technical competence. There also remain some deeper theoretical mysteries which are not so simple to explain. Here is a list of some of these items, posed as problems for the reader.

(1) Derive the asymptotic behavior (1.35) and explain the meaning of the torsion factor in (1.36). Use this reconcile the extra factor of $\sqrt{2}$ in (4.2) and (4.5). Also, explain the factor of $\sqrt{2 \pi^{2}}$ in (1.34).

(2) Derive (2.20) directly from (2.19).

(3) Give a direct proof using Witten's formalism of the Kirby-Melvin periodicity noted after (2.28).

(4) Prove the error estimate needed in Appendix A.

(5) Prove (4.3) directly from (2.21) (with $q= \pm 1$ ).

Our goal is these computer calculations was to document assertions about the Chern-Simons theory derived from the path integral. We feel that our results in Sect. 4 provide sufficient grounds for believing that this quantum field theory works as advertised. (The unresolved details itemized above are simply that unresolved details. They do not invalidate this belief.) While some aspects of the theory are understood mathematically from a different point of view, we are unaware of significant mathematical understanding that proceeds directly from the path integral. This, then, is the geometer's task: Explain the Chern-Simons theory in terms which are as simple and direct as the path integral. There should be a common framework which includes both the exact solution and the asymptotic formula. One could try to directly construct a measure and so the path integral. Such an approach works in case we replace $G=S U(2)$ by a finite group [FQ]. But this only involves finite measures, so eliminates many features of the problem. Still, geometers and topologists are familiar with many forms of "integration" which do not involve measures directly, and we are hopeful that some of these tools can be brought to bear on topological quantum field theory.

\section{Appendix A: Large $k$ Asymptotics for $L(p, \pm 1)$}

We provide a partial proof of the following asymptotic estimate using a "discrete stationary phase approximation."

Proposition A.1. As $k \rightarrow \infty$,

$$
\sum_{\alpha=1}^{k} \sin ^{2}\left(\frac{\alpha \pi}{k}\right) e^{\mp 2 \pi i\left(\frac{\alpha^{2} p}{4 k}\right)} \sim \sqrt{\frac{2 k}{p}} e^{\mp \pi i / 4} \sum_{n=1}^{\left[\frac{p-1}{2}\right]} \sin ^{2}\left(\frac{2 n \pi}{p}\right) e^{ \pm 2 \pi i\left(\frac{n^{2} k}{p}\right)} .
$$


The asymptotic formula (4.2) follows easily from this result. Our proof lacks a certain error estimate.

Proof. For simplicity we only consider the top sign in (A.2). Set,

$$
\alpha_{n}(k)=\frac{2 n k}{p}, \quad n=0,1,2, \ldots,\left[\frac{p}{2}\right],
$$

and split the region of summation on the left-hand side of (A.2) into intervals

$$
\alpha_{n}(k)-c k^{v} \leqq \alpha \leqq \alpha_{n}(k)+c k^{v}
$$

and intervals

$$
\alpha_{n-1}(k)+c k^{v} \leqq \alpha \leqq \alpha_{n}(k)-c k^{v},
$$

where $c$ is any positive constant and $v$ is a fixed number $\frac{1}{2}<v<\frac{3}{4}$. Consider first the integral (A.3) for $n$ fixed. Write

$$
\alpha_{n}(k)=x_{n}(k)+\varepsilon_{n}(k), \quad x_{n} \in \mathbb{Z}, 0 \leqq \varepsilon_{n}(k)<1,
$$

and let $j$ be the integer variable defined by

$$
\alpha=x_{n}(k)+j=\alpha_{n}(k)+j-\varepsilon_{n}(k) .
$$

Then after some simplification, the summation over the interval (A.3) is

$$
\sum_{-c k^{\nu} \leqq j \leqq c k^{\nu}} \sin ^{2}\left(\frac{2 n \pi}{p}+\frac{j \pi}{k}-\frac{\varepsilon_{n}(k) \pi}{k}\right) e^{-2 \pi i\left(\frac{n^{2} k}{p}\right)} e^{2 \pi i n \varepsilon_{n}(k)} e^{-2 \pi i\left(\frac{\left(j-\varepsilon_{n}(k)\right)^{2} p}{4 k}\right)} .
$$

The estimates $\sin ^{2}(x+\varepsilon)=\sin ^{2} x+O(\varepsilon)$ and $e^{i(x+\varepsilon)}=e^{i x}+O(\varepsilon)$ show that (A.5) and

$$
\sin ^{2}\left(\frac{2 n \pi}{p}\right) e^{2 \pi i\left(\frac{n^{2} k}{p}\right)} \sum_{-c k^{\nu} \leqq j \leqq c k^{\nu}} e^{-2 \pi i\left(\frac{j^{2} p}{4 k}\right)} .
$$

differ by a term of order $O\left(k^{2 v-1}\right)$. Using (A.7) below we see that the ratio of (A.5) to (A.6) differs from 1 by $O\left(k^{2 v-3 / 2}\right)$, which tends to zero as $k \rightarrow \infty$. We claim that (A.6) is asymptotic to an integral as $k \rightarrow \infty$ :

$$
\sum_{-c k v \leqq j \leqq c k^{\nu}} e^{-2 \pi i\left(\frac{j}{\sqrt{k}}\right)^{2} \frac{p}{4}} \sim \sqrt{k} \int_{-\infty}^{\infty} d x e^{-2 \pi i\left(\frac{x^{2} p}{4}\right)} .
$$

To prove (A.7) notice that on the interval $\left[\frac{j}{\sqrt{k}}, \frac{j+1}{\sqrt{k}}\right]$ we have the easy estimate

from the derivative. Thus

$$
\begin{aligned}
\left|e^{-2 \pi i\left(\frac{x^{2} p}{4}\right)}-e^{-2 \pi i\left(\frac{j}{\sqrt{k}}\right)^{2} \frac{p}{4}}\right| & \leqq \pi p \frac{|j|+1}{\sqrt{k}} \frac{1}{\sqrt{k}}, \\
& \leqq c \frac{|j|}{k}
\end{aligned}
$$

$$
\int_{\frac{j}{\sqrt{k}}}^{\frac{j+1}{\sqrt{k}}} d x e^{-2 \pi i\left(\frac{x^{2} p}{4}\right)}-\frac{1}{\sqrt{k}} e^{-2 \pi i \frac{j}{\sqrt{k}} 2 \frac{p}{4}} \leqq c \frac{|j|}{k^{3 / 2}} .
$$


Summing over $-c k^{v} \leqq j \leqq c k^{v}$, and applying the triangle inequality, we conclude

$$
\begin{aligned}
\left|\int_{-c k^{\nu-1 / 2}}^{c k^{\nu-1 / 2}} d x e^{-2 \pi i\left(\frac{x^{2} p}{4}\right)}-\sum_{-c k^{\nu} \leqq j \leqq c k^{\nu}} \frac{1}{\sqrt{k}} e^{-2 \pi i\left(\frac{j}{\sqrt{k}}\right)^{2} \frac{p}{4}}\right| & \leqq c \frac{\left(k^{\nu}\right)^{2}}{k^{3 / 2}} \\
& =c k^{2 v-3 / 2} .
\end{aligned}
$$

The desired assertion (A.7) follows by taking $k \rightarrow \infty$ in (A.8). We explicitly evaluate the integral in (A.7) as

$$
\int_{-\infty}^{\infty} d x e^{-2 \pi i\left(\frac{x^{2} p}{4}\right)}=\sqrt{\frac{2}{p}} e^{-\pi i / 4}
$$

Combining (A.6), (A.7), and (A.9) we see that the asymptotic contribution of the interval (A.3) is

$$
\sqrt{\frac{2 k}{p}} e^{-\pi i / 4} \sin ^{2}\left(\frac{2 n \pi}{p}\right) e^{2 \pi i\left(\frac{n^{2} k}{p}\right)} .
$$

The sum over $n$ is the right-hand side of (A.2).

Therefore, it remains to show that the sum over each interval (A.4) is smaller than $O\left(k^{1 / 2}\right)$ as $k \rightarrow \infty$. This is the error estimate we cannot provide.

\section{Appendix B: Twisting a 2-Framing}

Let $M$ be a 2-framed 3-manifold containing a solid torus $D^{2} \times S^{1}$. Suppose we remove the solid torus and reglue it after applying the Dehn twist $T$ given by (1.6). The resulting manifold is still diffeomorphic to $M$. In this appendix we verify that the resulting 2-framing on $M$ differs from the original one by a generator of $\pi_{3}(\operatorname{Spin}(6)) \cong \mathbb{Z}$.

This is basically a local computation. Since Spin(6) is 2-connected, we may isotope the 2-framing of $M$ so that on $D^{2} \times S^{1}$ it agree with a standard model (which we specify below). The model will agree with the stable product 2-framing on $\partial D^{2} \times S^{1}$. When we apply the Dehn twist $T$, the only effect on this 2 -framing on $\partial D^{2} \times S^{1}$ will be a shear in the vectors pointing in the $S^{1}$ direction. This shear should be canonically straightened to obtain the new 2-framing on $M$. It suffices to examine the change of framing in the model over $D^{2} \times S^{1}$. Furthermore, the effect of the twist on the vectors in the $S^{1}$ direction is trivial, so we will destabilize and work with the Spin(4) bundle tangent to the $D^{2}$ fibers.

We construct the model as follows: Let $D^{2}$ be the unit disk in $\mathbb{C}$, $D^{2}=\left\{r e^{i \theta} \mid 0 \leqq r \leqq 1\right\}$. Identify twice the tangent bundle of $D^{2}$ with $D^{2} \times \mathbb{H}$, where $\mathbb{H}=\mathbb{C} \oplus \mathbb{C} j$ is the quaternions. Thus the trivial 2-framing of $D^{2}$ is given by $\alpha=(1, i, j, k)$. Let $L_{q}$ denote left multiplication by $q$ in $\mathbb{H}$. Then the stable product 2 -framing on $\partial D^{2}$ is given by $L_{e^{i \theta}} \alpha$. Let $\lambda:[0,1] \rightarrow \mathbb{R}$ be a smooth monotonic function with $\lambda=0$ near 0 and $\lambda=\pi / 2$ near 1 . Let $f: D^{2} \rightarrow S^{3} C \mathbb{H}$ be the function

$$
f(r, \theta)=e^{i \theta / 2} e^{j \lambda(r)} e^{-i \theta / 2} e^{-j \lambda(r)} .
$$

Then $f$ is well defined $(f(r, \theta+2 \pi)=f(r, \theta))$ with $f(r, \theta)$ equal to 1 near 0 , and equal to $e^{i \theta}$ near $\partial D$. Thus, $L_{f(r, \theta)^{\alpha}}$ is a 2 -framing of $D^{2}$ which restricts to the product 2-framing on $\partial D^{2}$. Applying this to each $D^{2} C D^{2} \times S^{1}$ yields the model 2-framing of $D^{2} \times S^{1}$. 
The Dehn twist is given by

$$
\begin{aligned}
\tau: D^{2} \times S^{1} & \rightarrow D^{2} \times S^{1} \\
(r, \theta, \varphi) & \mapsto(r, \theta+\varphi, \varphi) .
\end{aligned}
$$

The new 2-framing is given by $L_{\left(\tau_{*} f\right)(r, \varphi)} \alpha$, where $\left(\tau_{*} f\right)(r, \varphi)=e^{i \varphi} f(r, \theta-\varphi)$. The 2-framings differ by the function $g: D^{2} \times S^{1} \rightarrow S^{3}$ defined by

$$
\begin{aligned}
g(r, \theta, \varphi) & =e^{i \varphi} f(r, \theta-\varphi)(f(r, \theta))^{-1} \\
& =e^{i(\theta+\varphi) / 2} e^{j \lambda(r)} e^{i \varphi / 2} e^{-j \lambda(r)} e^{-i \theta / 2} .
\end{aligned}
$$

Note that $g(r, \theta, \varphi)=1$ for $r=1$ or $\varphi=0$, so we get a well-defined element $[g] \in \pi_{3}\left(S^{3}\right)$. The composition

$$
S^{3}=S p(1) \times p t \subset S p(1) \times S p(1)=\operatorname{Spin}(4) \subset \operatorname{Spin}(6)
$$

induces an isomorphism on $\pi_{3}$, yielding the desired element $[g] \in \pi_{3}(\operatorname{Spin}(6))$, so it suffices to show that $g: D^{2} \times S^{1} \rightarrow S^{3}$ is a map of degree \pm 1 . But $j$ is a regular value of $g$ whose preimage is a single point. Specifically, suppose $g(r, \theta, \varphi) \in \mathbb{C} j$. Then $z \in \mathbb{C} j$, where

$$
z=e^{j \lambda(r)} e^{i \varphi / 2} e^{-j \lambda(r)} .
$$

Since $\operatorname{Re} z=0=\cos (\varphi / 2)$ we have $\varphi=\pi$ and

$$
z=i e^{-2 j \lambda(r)} \in \mathbb{C} j \text {. }
$$

Thus $\lambda(r)=\pi / 4$ (determining $r$ ) and $g(r, \theta, \varphi)=e^{i \theta} j$ (determining $\theta=0$ ). It is easily checked that $d g$ is onto at this point.

\section{References}

[A1] Atiyah, M.F.: On framings of 3-manifolds. Topology 29, 1-7 (1990)

[A2] Atiyah, M.F.: Topological quantum field theory. Publ. Math. Inst. Hautes Etudes Sci. (Paris) 68, 175-186 (1989)

[APS] Atiyah, M.F., Patodi, V.K., Singer, I.M.: Spectral asymmetry and Riemannian geometry. I. Math. Proc. Cambridge Philos. Soc. 77, 43-69 (1975); II. Math. Proc. Cambridge Philos. Soc. 78, 405-432 (1975); III. Math. Proc. Cambridge Philos. Soc. 79, 71-99 (1976)

[C] Cheeger, J.: Analytic torsion and the heat equation. Ann. Math. 109, 259-322 (1979)

[DW] Dijkgraaf, R., Witten, E.: Topological gauge theories and group cohomology. Commun. Math. Phys. 129, 393-429 (1990)

[F1] Freed, D.S.: Classical Chern-Simons theory. Preprint

[F2] Freed, D.S.: Reidemeister torsion, spectral sequences, and Brieskorn spheres. Preprint

[Fl] Floer, A.: An instanton-invariant for 3-manifolds. Commun. Math. Phys. 118, 215-240 (1988)

[FS] Fintushel, R., Stern, R.: Instanton homology of Seifert fibered homology three spheres. Proc. Lond. Math. Soc. 61, 109-137 (1990)

[FQ] Freed, D.S., Quinn, F.: Chern-Simons theory with finite gauge group. Preprint

[Fr] Franz, W.: Über die Torsion einer Überdeckung. J. Reine Angew. Math. 173, 245-254 (1935)

[J] Jeffrey, L.: On some aspects of Chern-Simons theory. Oxford Univ. D. Phil. thesis. In preparation

[K] Kirby, R.: A calculus for framed links in $S^{3}$. Invent. Math. 45, 35-56 (1978)

[KK] Kirk, P., Klassen, E.: Chern-Simons invariants of 3-manifolds and representation spaces of knot groups. Math. Ann. 287, 343-367 (1990) 
[KM1] Kirby, R., Melvin, P.: Evaluations of the 3-manifold invariants of Witten and Reshetikhin-Turaev for $\mathfrak{s l}(2, \mathbb{C})$. Geometry of low-dimensional manifolds: Donaldson, S.K., Thomas, C.B. (eds.). London Mathematical Society Lecture Note Series, Vol.151, pp. 101-114. Cambridge: Cambridge University Press 1990

[KM2] Kirby, R., Melvin, P.: On the 3-manifold invariants of Witten and Reshetikhin-Turaev for $\mathfrak{s l}(2, \mathbb{C})$. Preprint

[Ka] Kaplan, S.: Constructing framed 4-manifolds with given almost framed boundaries. Trans. AMS 254, 237-263 (1979)

[Mi] Milnor, J.: Whitehead torsion. Bull. Am. Math. Soc. 72, 358-426 (1966)

[Mu] Muller, W.: Analytic torsion and R-torsion of Riemannian manifolds. Adv. Math. 28, 233-305 (1978)

[R] Rolfson, D.: Knots and Links. Berkeley: Publish or Perish 1976

[Re] Reidemeister, K.: Homotopieringe und Linsenräume. Hamburger Abhandl. 11, 102-109 (1935)

[RS1] Ray, D.B., Singer, I.M.: R-torsion and the laplacian on Riemannian manifolds. Adv. Math. 7, 145-210 (1971)

[RS2] Ray, D.B., Singer, I.M.: Analytic torsion. Partial Differential Equations, Proceedings of Symposia in Pure Mathematics, Vol.23, pp.167-182. American Mathematical Society 1973

[RT] Reshetikhin, N.Yu., Turaev, V.G.: Invariants of 3-manifolds via link polynomials and quantum groups. Invent. Math.

[SZ] Skoruppa, N., Zagier, D.: A trace formula for Jacobi forms. J. Reine Angew. Math. 393, 168-198 (1989)

[V] Verlinde, E.: Fusion rules and modular transformations in $2 d$ conformal field theory. Nucl. Phys. B 300, 360 (1988)

[W] Witten, E.: Quantum field theory and the Jones polynomial. Commun. Math. Phys. 121, 351 (1989)

[Wa] Walker, K.: On Witten's 3-manifold invariants. Preprint

[Wo] Wolfram, S.: Mathematica. Reading, MA: Addison-Wesley 1988

Communicated by A. Jaffe

Note added in proof. We thank Don Zagier for bringing to our attention an error in Appendix A, which we have corrected. Note that the sum in (A.2) can be evaluated explicitly using the results of Lisa Jeffery [J], which we did in (4.4). The sum can also be evaluated using work of Skoruppa and Zagier [SZ]. Of course. These explicit evaluations prove Proposition A.1 
